\title{
LncGBP9/miR-34a axis drives macrophages toward a phenotype conducive for spinal cord injury repair via STAT1/STAT6 and SOCS3
}

Jiahui Zhou ( $\nabla$ zjh5518@sina.com )

Central South University Third Xiangya Hospital

\section{Zhiyue Li}

Central South University Third Xiangya Hospital

Tianding Wu

Central South University Third Xiangya Hospital

Qun Zhao

Central South University Third Xiangya Hospital

Qiancheng Zhao

Central South University Third Xiangya Hospital

\section{Yong Cao}

Central South University Third Xiangya Hospital

\section{Research}

Keywords: spinal cord injury (SCI), macrophage polarization, IncGBP9, SOCS3, STAT1/STAT6, miR-34a

Posted Date: February 26th, 2020

DOI: https://doi.org/10.21203/rs.2.19759/v2

License: (9) (i) This work is licensed under a Creative Commons Attribution 4.0 International License. Read Full License

Version of Record: A version of this preprint was published at Journal of Neuroinflammation on April 28th, 2020. See the published version at https://doi.org/10.1186/s12974-020-01805-5. 


\section{Abstract}

Background: Acute spinal cord injury (SCl) could cause mainly two types of pathological sequelae, the primary mechanical injury, and the secondary injury. The inappropriate activation of macrophage phenotypes (macrophage M1 or M2 polarization) might cause the failure to post-SCl repair.

Methods: SCl model was established in Balb/c mice and the changes in macrophage phenotypes after $\mathrm{SCl}$ was monitored. Bioinformatic analyses were performed to select factors that might regulate macrophage polarization after SCl. Mouse bone marrow-derived macrophages (BMDMs) were isolated, identified, and induced for M1 or M2 polarization; the effects of IncRNA Inc GBP9 (guanylate binding protein-9) and SOCS 3 (suppressor of cytokine signaling 3) on macrophages polarization were examined in vitro and in vivo. The predicted miR-34a binding to IncGBP9 and SOCS3 was validated; the dynamic effects of IncGBP9 and miR-34a on SOCS3, STAT 1 (signal transducer and activator of transcription 1)/STAT6 (signal transducer and activator of transcription 6) signaling, and macrophage polarization were examined. Finally, we investigated whether STAT6 could bind the miR-34a promoter to activate its transcription.

Results: In SCI Balb/c mice, inappropriate activation of macrophage phenotypes was observed after SCl. In M1 macrophages, IncGBP9 silencing significantly decreased p-STAT1 and SOCS3 expression and protein levels, as well as the production of IL-6 (Interleukin 6) and IL-12 (Interleukin 12); in M2 macrophages, IncGBP9 overexpression increased SOCS3 expression and protein levels while suppressed p-STAT6 levels and the production of IL-10 (Interleukin 10) and TGF- $\beta 1$ (transforming growth factor beta 1), indicating that IncGBP9 overexpression promotes the M1 polarization of macrophages. In IncGBP9silenced $\mathrm{SCl}$ mice, the $\mathrm{M} 2$ polarization was promoted on day 28 after operation, further indicating that IncGBP9 silencing revised the predominance of M1 polarization at the late stage of secondary injury after $\mathrm{SCl}$, therefore improving the repair after SCl. IncGBP9 competed with SOCS3 for miR-34a binding to counteract miR-34a-mediated suppression on SOCS3 and then modulated STAT1/STAT6 signaling and the polarization of macrophages. STAT6 bound the promoter of miR-34a to activate its transcription.

Conclusions: In macrophages, IncGBP9 sponges miR-34a to rescue SOCS3 expression, therefore modulating macrophage polarization through STAT1/STAT6 signaling. STAT6 bound the promoter of miR-34a to activate its transcription, therefore forming two different regulatory loops to modulate the polarization of macrophages after $\mathrm{SCl}$.

\section{Background}

Spinal cord injury (SCl) is one of the most important global contributors to disability and related death; however, the treatment efficacy of $\mathrm{SCl}$ is still unsatisfactory [1-3]. Acute $\mathrm{SCl}$ could cause mainly two types of pathological sequelae, the primary mechanical injury and the secondary injury [4]. Direct mechanical trauma could cause the primary SCl, followed by the secondary injury through activating several pathophysiological processes, such as inflammation, dysregulation of microvascular perfusion, 
deregulated generation of free radicals, dysregulation of cell apoptosis, and broken ionic homeostasis [59], which would directly lead to the destruction of intact axonal tracts and hinder the structural and functional recoveries after initial SCl.

During both the acute and chronic phases of the secondary injury when the central nervous system (CNS) evokes innate and adaptive immunity $[9,10]$, inflammatory responses have been regarded as the central issue. Recent studies have shown that the sequential activation of immune cells, including resident and recruited subtypes, may play an important role in the secondary inflammatory/immune responses after CNS injury, including SCl. The macrophage is a critical cell type in the innate immune response within CNS. Macrophages are heterogeneous and comprised predominantly of two groups: specialized CNSresident microglia-derived macrophages and bone marrow-derived macrophages (BMDMs). Microglial cells are renewed by local proliferation, arrive in the CNS from yolk sacks in development and are responsible for surveying the CNS parenchyma and aid in synaptic pruning $[11,12]$. Resident microglia are located in immunizing CNS tissues [11]. BMDMs are often localized mainly in the margins of the lesion site following $\mathrm{SCl}$, while the resident microglia-derived macrophages are usually distributed in the lesion core and its margins. After injury, infiltrating BMDMs migrate to the epicenter of injury, while microglia-derived macrophages localize to the edges of lesion [13]. In other words, the majority of macrophages in the lesion site are BMDMs rather than locally activated microglia. These two populations of macrophages with different locations have different functions. Residential microglia-derived macrophages form a border that seems to seal the lesion and block the spread of damage, whereas BMDMs enter the epicenter of injured spinal cord and phagocytize apoptotic and necrotic cells and clear tissue debris such as myelin debris [14].

Macrophages are phenotypically dynamic in both morphology and function, ranging from resting ramified steady-state (M0) to a pro-inflammatory (M1) or an anti-inflammatory phenotype (M2) [15]. In fact, the situation might be more complicated in vivo. After $\mathrm{SCl}$, the collective actions of the non-specific and adaptive immune system can be recruited and serve various functions which are both neurotoxic and neuroprotective. The interferon- $\gamma$ (IFN- $\gamma$ ) and prototypical T-helper 1 cytokine (TH1) can activate and induce macrophages to produce cytotoxic mediators (reactive oxygen and nitrogen species) and proinflammatory cytokines (iNOS (inducible nitric oxide synthase), IFN- $\gamma$, TNF-a (tumor necrosis factor alpha), CCL5 (C-C motif chemokine ligand 5), IL-23 (Interleukin 23), IL-12, IL-6 and IL-1ß), and increase their ability to kill pathogens within cells. By contrast, the IL-13, IL-4, TH2 and so on inhibit macrophages from producing pro-inflammation cytokines $[16,17]$, and increase their ability to kill extracellular pathogens such as parasite infection $[18,19]$. These two different macrophage phenotypes induced by either TH1 (IFN- $y$ and TLR signaling) or TH2 (IL-13 and IL-4) are known as M1 or 'classically activated' macrophage, while the latter is called M2 or 'alternatively activated' macrophage [20]. Within a few hours after $\mathrm{SCl}$, macrophages first polarized into M1 macrophages in response to IFN- $\gamma$, LPS (Lipopolysaccharides), TNF-a, and other stimuli, and reaches a peak on day 1 after SCl. Macrophage surface Toll-like receptors (TLRs) are activated and then induce the recruitment of downstream protein myeloid differentiation factor 88 (MyD88), the activation of the downstream pathways, including NF-KB (nuclear factor kappa B), JAK (Janus kinase)-STAT (signal transducer and activator of transcription), JNK 
(c-Jun N-terminal kinases), MAPK (mitogen-activated protein kinase), and PI3K (Phosphoinositide 3kinases)/Akt (protein kinase B, PKB) [21], and promote the polarized macrophages to release TNF-a, IL- $\beta$, IL-6, other inflammatory factors [22] and chemokines (CCL8 and CCL9), and cyclooxygenase (Cox), which in turn promote the differentiation of more macrophages to M1. Later, the polarized M1 macrophages exhibit stronger phagocytosis and antigen-presenting ability. Moreover, a large number of M1 macrophage-secreted inflammatory cytokines, reactive oxygen species (ROS), reactive nitrogen (RNS), prostaglandin (PGE2) and other active substances cause damage to neurons and glia, leading to neuronal apoptosis [23]. As for M2 macrophages, cell surface receptors bind IL-4 and IL-13 to promote STAT6 phosphorylation, therefore stimulating the macrophage polarization into M2 type [24]. The markers of M2 macrophages include arginase (Arg-1), resistin-like molecules (Fizz-1), IL-10, TGF- $\beta$, mannitol receptors (including CD163, CD204, CD206), etc. [25]. M2 macrophages highly express IL-10, IL4 , IL-13, TGF- $\beta$, and neurotrophic factor (NTF), which can inhibit neuronal apoptosis and the proinflammatory effects of $\mathrm{M} 1$ macrophages, promoting nerve tissue repair. There are 3 subtypes of $\mathrm{M} 2$ macrophages including M2a/b/c; M2a macrophages appear on day 1 3 after SCl, highly express CD206 and Arg1 and exert anti-inflammatory and repair functions [26]; M2b appears on day 3 7 after SCl, express high IL-10 and low Arg1; M2c appears the latest and could inhibit the production of inflammatory cytokines and inflammation [27].

Macrophages are widely malleable in functions, allowing them to convert from one phenotype to another under the broad stimuli in the post-SCl inflammatory microenvironment. M1 macrophages play a detrimental role after $\mathrm{SCl}$, while $\mathrm{M} 2$ macrophages play a promotive role in the regenerative growth responses in adult sensory axons. After $\mathrm{SCl}$, increased macrophages polarization toward $\mathrm{M} 1$ phenotype and the decreased number of M2 macrophages may lead to or even aggravate the secondary injury. Previously, it has been reported that the angiotensin-converting enzyme (ACE)-C domain overexpression in macrophages would result in the transformation of macrophages toward M1 phenotype under tumor stimulation, accompanied by enhanced activation of NF-KB and signal transducer and activator of transcription 1 (STAT1) and attenuated activation of STAT3/6 [28]. Yao et al. reported that the M1 polarization could be hindered by a crucial immune inhibitory receptor, namely programmed cell death 1 (PD-1), via suppressing the phosphorylation of STAT1; in the meantime, PD-1 also promoted the M2 polarization via enhancing the phosphorylation of STAT6 [29]. Consistently, the M1 polarization was enhanced by activating STAT1 and NF-KB in PD-1 knockout mice [29]. Therefore, investigating the factors and mechanisms of regulating STAT1/STAT6 signaling may help to understand the mechanism of M1/2 macrophage polarization switch.

During the past decades, non-protein coding RNAs (ncRNAs) have been regarded as key regulators by playing diverse roles in not only fundamental biological but also pathological processes [30,31]. Among them, microRNA (miRNA) and long noncoding RNAs (IncRNAs) are the most well-known. miRNAs induce either mRNA degradation or block mRNA translation depending on the complete or incomplete complementarity [32], while IncRNAs could serve as competing endogenous RNAs (ceRNAs) to counteract miRNA-mediated inhibition on miRNA downstream transcripts, therefore exerting their biological functions [33-35]. Like miRNA, there is new evidence that IncRNAs might be a novel type of regulator 
macrophage immune response [36]. LncRNAs have been reported to be partially responsible for the gene expression dysregulation during macrophage polarization [37]. Huang et al. [38] also identified differentially-expressed IncRNAs in M1 or M2 polarized macrophages. Of these deregulated IncRNAs, IncRNA TCONS_00019715 has higher expression in M1 macrophages than that in M2 macrophages. When proinflammatory macrophages convert to anti-inflammatory macrophages, TCONS_00019715 expression decreases. However, it increases when anti-inflammatory macrophages convert to proinflammatory phenotype. Knockdown of TCONS_00019715 diminishes the expression of proinflammatory macrophage markers and increases the expression of anti-inflammatory markers. TCONS_00019715 promotes macrophage transition to proinflammatory macrophages by downregulating PAK1 (P21 (RAC1) activated kinase 1), an important regulator of cytoskeletal remodeling and cell motility in mononuclear phagocytic system [38]. Another IncRNA, IncRNA E330013P06, was found to regulate proinflammatory gene expression and foam cell formation in macrophages [39]. Based on these previous findings, we hypothesize that IncRNAs may participate in macrophage polarization by regulating related molecules and STAT1/STAT6 signaling pathways, most possibly in a miRNA-dependent manner.

In the present study, we conducted the SCI model in Balb/c mice and examined the expression of M1/2 macrophage markers on day $1,3,7,14$, and 28 after the operation to monitor the changes in the macrophage polarization. Next, by downloading and analyzing online microarray profiles reporting differentially-expressed IncRNAs and genes in M1/2 macrophages, we selected IncRNAs and genes related to macrophage polarization, namely IncGBP9 in mice and SOCS3 (suppressor of cytokine signaling 3). Mouse bone marrow-derived macrophages (BMDMs) were isolated, identified, induced to differentiate into M1/2 macrophages, and examined for expression of SOCS3, STAT1 and STAT6 and cytokine production. Next, the effects of IncGBP9 on macrophage polarization, SOCS3 expression, and STAT1/STAT6 signaling were evaluated in vitro and in vivo. Since miR-34a has been reported to promote the M2 macrophage polarization [40] and be predicted to target IncGBP9 and SOCS3, we further investigated whether IncGBP9 could compete with SOCS3 for miR-34a binding, thereby counteracting miR-34a-mediated SOCS3 suppression. The predicted bindings of miR-34a to IncGBP9 and SOCS3 were validated and the dynamic effects of IncGBP9 and miR-34a on SOCS3, STAT1/STAT6 signaling, and macrophage polarization were examined. Finally, we investigated whether STAT6 could bind the miR-34a promoter to activate its transcription. In summary, we provide a novel mechanism by which the IncGBP9/miR-34a axis modulates STAT1/STAT6 to affect macrophage polarization via SOCS3.

\section{Methods}

\section{Spinal cord injury (SCl) model in Balb/c mice}

Balb/c mice (The SLAC experimental animal center, Shanghai, China) received a moderate midthoracic (T9-10) SCl as described previously [41]. Sham mice received a laminectomy without SCl. To identify the $\mathrm{SCl}$ model in mice, tissue in lesion epicenter was collected $1,3,7,14$, or $28 \mathrm{~d}$ after SCl and examined for the M1 macrophage marker CD16/32 and M2 macrophage marker Arg1 by immunofluorescence (IF) staining, the mRNA expression of M1 macrophage markers iNOS, CD16/32, and IFN-y and M2 
macrophage markers Arg1, CD206, and IL-4 by real-time PCR and Immunoblotting. Locomotor recovery of mice was assessed by two persons using the Basso Mouse Scale (BMS) [42] open field test at 1, 3, 7, 14, and $28 \mathrm{~d}$ after injury following the methods described previously $[42,43]$. All procedures involving animals were approved by the Central South University Research Ethics Committee.

\section{Isolation and identification of mouse bone marrow-derived macrophages}

Bone marrow-derived macrophages (BMDMs) were isolated from the bilateral femurs and tibias of adult Balb/c mice and then cultured as described previously [44, 45]. 20\% supernatant from L929 cells was added to stimulate BMDMs to differentiate into macrophages for 7 days [46].

\section{Induction and identification of M0 macrophages polarized toward M1/2 macrophages}

The induction of M0 macrophages differentiation into M1 or M2 macrophages was conducted with 100 $\mathrm{ng} / \mathrm{ml}$ LPS (Sigma-Aldrich) $+20 \mathrm{ng} / \mathrm{ml} \mathrm{IFN- \gamma} \mathrm{(eBioscience)} \mathrm{or} 20 \mathrm{ng} / \mathrm{ml} \mathrm{IL-4} \mathrm{(eBioscience),} \mathrm{respectively,}$ for $48 \mathrm{~h}$ following the methods described previously [44-46].

\section{Recombinant adenoviruses preparation, transduction and injection}

The recombinant adenoviruses expressing IncGBP9 shRNA or IncGBP9-overexpressing fragment or scramble RNA (NC shRNA) were generated using the AdEasyTM Vector System (Invitrogen) following the methods described before [47]. For in vitro experiments, adv-sh-NC, adv-sh-IncGBP9, adv-NC and advIncGBP9 were then diluted in PBS and administered at a concentration of $2 \times 10^{7}$ pfu/well in 12-well plate. $24 \mathrm{~h}$ later, M0 macrophage were stimulated to polarization. For in vivo experiments, total $1 \mu \mathrm{l}$ of adv-sh-NC or adv-sh-IncGBP9 $\left(1 \times 10^{10} \mathrm{pfu} / \mathrm{ml}, \mathrm{n}=8\right)$ was injected to the injured spinal cord at a depth of $0.5 \mathrm{~mm}$ and $1 \mathrm{~mm}$ (each depth $0.5 \mu \mathrm{l}$ ) using 5 - $\mu \mathrm{l}$ Hamilton syringe, each injection was performed at 0.2 $\mu \mathrm{l} / \mathrm{min}$. Before withdraw the syringe, the needle left in place for a further $2 \mathrm{~min}$ to avoid the viral leakage. The dorsal muscle and skin were then sutured. 28 days later, the mice were sacrificed for further experiments.

\section{Immunofluorescence staining}

Spine cord tissues were fixed in $4 \%$ paraformaldehyde and dehydrated using $30 \%$ sucrose overnight. After embedding into OCT compound (Tissue Tek), tissues were cut into $16 \mu \mathrm{m}$ section. Sections were blocked using $5 \%$ normal goat serum and then were incubated with the diluted primary antibody specific to F4/80 (ab111101, abcam, MA, USA), CD16/32 (Catalog \# 14-0161-82, Invitrogen, Waltham, MA, USA) and Arg1 (sc-271430, Santa Cruz, USA), overnight at $4^{\circ} \mathrm{C}$. Cy3 or FITC-conjugated secondary antibody (Santa Cruz) were incubated with sections at room temperature for $1 \mathrm{~h}$. For cellular immunofluorescence, cells were fixed with $4 \%$ paraformaldehyde and incubated with primary antibody specific to F4/80 and CD11b (ab8878) and then incubated with Cy3 or FITC-conjugated secondary antibodies. DAPI (C1002, Beyotime, China) was used to stain the nucleus in tissue sections and cells before capturing images. The images were acquired using a fluorescence microscope (Nikon, Japan). 


\section{Immunoblotting}

Immunoblotting was performed as previous describe [48] using the following primary antibodies: antiiNOS (ab15323, Abcam,), anti-CD16 (ab203883)), anti-IFNy (500-P119, PeproTech, Rocky Hill, NJ, USA), anti-Arg1 (sc-271430, santa cruz), anti-CD206 (ab64693, abcam), anti-IL-4 (ab11524, Abcam), anti-STAT1 (ab3987, Abcam), anti-p-STAT1 (ab30645, Abcam), anti-STAT6 (ab32520, Abcam), anti-p-STAT6 (ab28829, Abcam), anti-SOCS3 (ab16030, Abcam), and the HRP-conjugated secondary antibody (1:5000). The plots were visualized by ECL Plus (Thermo).

\section{Real-time PCR}

Total RNA was extracted from target cells by using Trizol reagent (Invitrogen) and the expression of miRNA or mRNA was examined following the methods previously described [49] using a Hairpin-it TM miRNAs qPCR kit (Genepharma, Shanghai, China) or a SYBR Green PCR Master Mix (Qiagen), respectively. $\mathrm{U} 6$ or $\beta$-actin expression was used as an endogenous normalization, respectively. The threshold cycle $(\mathrm{Ct})$ was determined and relative mRNA and miRNA levels were calculated using $2^{-\Delta \Delta C t}$ methods. The primer sequences were listed in Table S1.

\section{ELISA}

Cell culture medium was collected for ELISA assay using human IL-6, IL-12, IL-10, and TGF $\beta 1$ ELISA kits according to the manufacturer's instructions (Santa Cruz Biotechnology, Santa Cruz, CA, USA) following the methods described previously [50]. The specific binding optical density was assayed immediately at $450 \mathrm{~nm}$ with a spectrophotometer (Bio-Rad Laboratories).

\section{Cell transfection}

Commercial SOCS3 overexpression vector, STAT6 overexpression vector, small interference RNA (si) for SOCS3 and STAT6, miR-34a mimics and inhibitor were obtained from RiboBio, Guangzhou, China. All transfections were performed with the help of Lipo2000 (Invitrogen) according to the manufacture's instruction. Twenty-four $\mathrm{h}$ after transfection, the M0 macrophages were polarized to M1/2 macrophages.

\section{Luciferase reporter assays}

For the validation of the binding of miR-34a to GBP9 or SOCS3 3'-UTR, the wild-type GBP9 or SOCS3 3'UTR luciferase reporter vector was constructed by cloning the fragment of GBP9 or SOCS3 3'-UTR to the Renilla psiCHECK2 vector (Promega, Madison, WI, USA) and named wt-GBP9/wt-SOCS3 3'-UTR; the mutant-type GBP9 or SOCS3 3'-UTR vector was constructed by mutating the predicted miR-34a binding site in GBP9 or SOCS3 3'-UTR and named mut-GBP9/mut-SOCS3 3'-UTR. These vectors were cotransfected in HEK293 cells with miR-34a mimics/inhibitor; For validation the binding of STAT6 to the promoter of miR-34a, wild- and mutant-type miR-34a promoter luciferase reporter vectors were constructed; STAT6 and wt- or mut-miR-34a promoter were then co-transfected in M0 macrophages followed by M2 polarization; the luciferase activity was detected using the Dual-Luciferase Reporter 
Assay System (Promega). The primers used for luciferase reporter vector construction were listed in Table S1.

\section{RNA Immunoprecipitation (RIP)}

RIP analysis was performed M1 macrophages using a Magna RIP RNA-Binding Protein Immunoprecipitation Kit (17-700, Millipore, Burlington, MA, USA) and a T7 High YieldRNA Synthesis Kit (E2040S, NEB, Ipswich, MA, USA) following the methods described previously [51]. The levels of IgG, GBP9 and miR-34a in the immunoprecipitates were measured by qRT-PCR. The primers were listed in the Table S1.

\section{Chromatin immunoprecipitation (ChIP)}

ChIP assays were performed following the methods described previously [52] using antibodies against STAT6 (ab32520, Abcam), a positive control antibody (RNA polymerase II), and a negative control nonimmune IgG. The immunoprecipitated DNA was cleaned, released, eluted, and used for downstream applications. The fold-enrichment (FE) was calculated as previously described [52].

\section{Statistical Analyses}

All the data of results from at least three independent experiments in the present study are first processed by SPSS17.0 (IBM, Armonk, NY, USA) and presented as the mean \pm S.D $n \geq 3$ independent experiments. The statistical comparison between means was conducted using a Student $t$-test where applicable. Differences among more than two groups were analyzed using one-way ANOVA. ${ }^{*} P<0.05 ; * \star P<0.01$.

\section{Results}

\section{Macrophage polarization during SCl}

To investigate the inappropriate activation of macrophage phenotypes (macrophage polarization) after $\mathrm{SCl}$, we established a mouse model of $\mathrm{SCl}$ using Balb/c mice following the methods described previously [41]. To identify the $\mathrm{SCl}$ model, we examined the content and distribution of macrophage marker F4/80, M1 macrophage marker CD16/32, and M2 macrophage marker Arg1 in spinal cords from sham group and $\mathrm{SCl}$ mouse on day $1,3,7,14,28$ after operation. As revealed by IF staining, compared to sham group, the fluorescence intensity representing F4/80 (red) and CD16/32 (green on the upper panel) gradually increased in a time-dependent manner in $\mathrm{SCl}$ group (Fig.1A); however, the fluorescence intensity representing Arg1 (green on the lower panel) increased moderately on day 1 and 3, reached a sharp peak value on day 7 , and then gradually decreased on day 14 and day 28 (Fig.1A). Consistently, the mRNA expression and the protein levels of M1 macrophage markers CD16, iNOS, and IFN- $\gamma$ and M2 macrophage markers Arg1, CD206, and IL-4 emerged similar trend. M1 macrophage markers increased after SCl in a time-dependent manner from day 1 to day 28 , while M2 macrophage markers increased moderately on day 1 and 3 , reached sharp peak values on day 7 , and then decreased gradually (Fig.1B-C). These data 
indicate that the inappropriate activation of macrophage phenotypes (macrophage polarization) exists after $\mathrm{SCl}$ and might participate in the dysfunction of $\mathrm{SCl}$ repair.

\section{Selection of IncRNAs and genes related to macrophage polarization}

As we have mentioned, the deregulation and dysfunction of IncRNAs in the process of a pro-inflammatory (M1) to an anti-inflammatory phenotype (M2) have been observed [38, 53]. In the present study, we attempted to identify IncRNAs related to the inappropriate activation of macrophage phenotypes (macrophage polarization) after SCl. The study downloaded and analyzed microarray profiles (GSE117040 and GSE5099) which reported upregulated IncRNAs in M1 macrophages. A total of 4 IncRNAs were reported to be upregulated in M1 macrophages by both profiles; there are homologous genes for IncRNA GBP1P1 (GBP9 in mice) and LINC00869 (Fam91a1 in mice) in mice (Fig. S1A).

Next, to further validate the involvement of these IncRNAs, we isolated BMDMs and identified them by examining macrophage markers F4/80 and CD11b using IF staining (Fig.2A). BMDMs (M0 macrophages) were then induced for differentiating towards M1 or M2 macrophages; the mRNA expression and protein levels of M1 macrophage markers iNOS and CD16 and M2 macrophage markers Arg1 and CD206 were examined to identify different subtypes. As shown in Fig.2B-C, iNOS and CD16 were significantly upregulated in M1 subtype while downregulated in M2 subtype; Arg1 and CD206 were remarkably upregulated in M2 subtype while downregulated in M1 subtype, indicating the successful induction. As revealed by real-time PCR, the expression of GBP9 and Fam91a1 were both significantly upregulated in M1 macrophages, GBP9 more upregulated (Fig.2D), indicating that GBP9 might be involved in macrophage M1/2 polarization. Reportedly, GBP1P1 is a pseudogene of the guanylatebinding protein of GB (guanylate-binding protein); this family is also involved in macrophage functions, such as IFN- $y$-mediated macrophage activation and immune defense [54]. More importantly, based on microarray profile or RNA-seq analyses GSE5099 (Fig.S1B), GSE117040 (Fig.S1C), E-GEOD-57494 (Fig.S1D), E-MTAB-2399 (Fig.S1E), and GSE40885 (Fig.S1F), IncRNA GBP1P1 is specifically highlyexpressed in human $\mathrm{M} 1$ macrophages and could be rapidly up-regulated after treatment with M1inducing factors LPS and IFN-y. Thus, GBP1P1 (IncGBP9 in mice) was selected for further experiments.

We then performed STRING (search tool for the retrieval of interacting genes/proteins) analyses on differentially-expressed genes in M1 macrophages reported previously [38, 55] to identify key regulators of the switch from a pro-inflammatory (M1) to an anti-inflammatory phenotype (M2). As revealed by STRING analyses, Tnf, SOCS3, and STAT1 are key factors in macrophage polarization (Fig.2E). Next, the mRNA expression and protein levels of M1-related STAT1 and p-STAT1, M2-related STAT6 and p-STAT6, and SOCS3 were examined in M0, M1, and M2 macrophages. As shown in Fig.2F and G, p-STAT1 and SOCS3 mRNA and protein levels were dramatically upregulated in M1 macrophages while p-STAT6 was upregulated in M2 macrophages. In the meantime, the production of STAT1 downstream cytokines, including IL-6 and IL-12 was increased in M1 macrophages while that of STAT6 downstream IL-10 and TGF- $\beta 1$ was increased in M2 macrophages (Fig. $2 \mathrm{H}$ ). These data indicate that IncGBP9 and SOCS3 expression are upregulated in M1 macrophages and might be related to M1/2 polarization. 


\section{Effects of IncGBP9 on macrophage polarization in vitro}

After selecting IncGBP9 for further experiments, we next evaluated its effects on macrophage polarization in vitro and in vivo. BMDMs were induced toward M0 macrophages for 7 days as described. Then, the silencing and overexpression of IncGBP9 was conducted in M0 macrophages for $48 \mathrm{~h}$ and the transduction was effective and confirmed by real-time PCR (Fig.3A). After transduction for $24 \mathrm{~h}$, LncGBP9-silenced and IncGBP9-overexpressing M0 macrophages were stimulated by LPS + IFN- $\gamma$ for M1 polarization. In M1 macrophages, IncGBP9 silencing or overexpression caused no significant changes in STAT1 mRNA expression; IncGBP9 silencing significantly downregulated, while IncGBP9 overexpression upregulated SOCS3 mRNA expression (Fig.3B). In M1 macrophages, the protein levels of p-STAT1 and SOCS3 were significantly reduced by IncGBP9 silencing while increased by IncGBP9 overexpression (Fig.3C); consistently, the production of IL-6 and IL-12 was also inhibited by IncGBP9 silencing while promoted by IncGBP9 overexpression in M1 macrophages (Fig.3D). On the contrary, IncGBP9overexpressing or IncGBP9-silenced macrophages were stimulated with IL-4 for M2 polarization. In M2 macrophages, IncGBP9 overexpression or silencing caused no significant changes in STAT6 mRNA expression; IncGBP9 overexpression significantly upregulated, while IncGBP9 silencing downregulated SOCS3 mRNA expression (Fig.3E). In M2 macrophages, IncGBP9 overexpression decreased p-STAT6 and increased SOCS3 protein, while IncGBP9 silencing increased p-STAT6 and decreased SOCS3 (Fig.3F); consistently, the production of IL-10 and TGF- $\beta 1$ was suppressed by IncGBP9 overexpression while promoted by IncGBP9 silencing in M2 macrophages (Fig.3G). These data indicate that IncGBP9 might modulate macrophage M1/2 polarization through affecting SOCS3 and the phosphorylation of STAT1/STAT6.

To further investigate the speculation, the study then co-transfected M0 macrophages with Adv-IncGBP9 and SOCS3-overexpressing vector (SOCS3 OE) or with Adv-IncGBP9 and si-SOCS3, induced transfected M0 macrophages toward M1 polarization, and examined for macrophage M1/2 polarization. In Adv-shIncGBP9 and SOCS3 OE co-transfected M1 macrophages, IncGBP9 silencing significantly inhibited SOCS3 mRNA expression (Fig.3H), decreased SOCS3 protein level and STAT1 phosphorylation (Fig.3I), and reduced the concentrations of IL-6 and IL-12 (Fig.3J). SOCS3 overexpression in M1 macrophages exerted opposite effects. The effects of IncGBP9 silencing on M1 macrophages were significantly reversed by SOCS3 overexpression. Next, M0 macrophages were co-transfected with Adv-IncGBP9 and siSOCS3, induced toward M2 polarization, and examined accordingly. In Adv-IncGBP9 and si-SOCS3 cotransfected M2 macrophages, IncGBP9 overexpression significantly upregulated SOCS3 mRNA expression (Fig.3K), increased SOCS3 protein level, inhibited STAT6 phosphorylation (Fig.3L), and reduced the concentrations of IL-10 and TGF $\beta$ (Fig.3M). SOCS3 silencing in M2 macrophages exerted opposite effects. The effects of IncGBP9 silencing on M2 macrophages were significantly reversed by sOCS3 silencing.

\section{Effects of IncGBP9 on macrophage polarization in vivo}


Next, the effects of IncGBP9 on macrophage polarization were evaluated in vivo. LncGBP9 silencing was conducted in the SCI mice model via injecting to the epicenter of injured spinal cord with Adv-sh-IncGBP9. At day 28 of SCl, Adv-sh-IncGBP9 effectively reduced the level of IncGBP9 in the injured spinal cords (Fig.4A). Next, we evaluated the BMS scores on day 1, 3, 7, 14, and 28 after the operation to access the effects of IncGBP9 silencing on SCl severity. As shown in Fig.4B, IncGBP9 silencing significantly reduced the BMS scores in $\mathrm{SCl}$ mice on day 14 and 28 after the operation, indicating IncGBP9 silencing in $\mathrm{SCl}$ mice promoted the repair after $\mathrm{SCl}$.

At the same time, the content and distribution of M1 macrophage marker CD16/32 and M2 macrophage marker Arg1 determined in IncGBP9-silenced SCI mice by IF staining and Immunoblotting on day 28 after the operation to investigate macrophage polarization. As shown in Fig.4C-D, the fluorescence intensity representing M1 marker CD16/32 was significantly inhibited in Adv-sh-IncGBP9-infected mice on day 28, while M2 marker Arg1 was increased in Adv-sh-IncGBP9-infected mice on day 28, compared to those in Adv-sh-NC group. Consistently, Fig.4E showed that the protein levels of p-STAT1 and SOCS3 were significantly decreased, while the protein levels of p-STAT6 were increased by IncGBP9 silencing in SCI mice on day 28 after the operation. These data indicate that IncGBP9 silencing might promote M2 and inhibit M1 polarization via STAT1/6 and SOCS3, therefore modulating the repair after SCI.

\section{LncGBP9 modulates SOCS3 through miR-34a in macrophages}

LncRNAs could serve as ceRNAs for miRNAs to counteract miRNA-mediated suppression on miRNA downstream transcripts, therefore exerting their biological functions [33-35]. Essandoh reported a number

of miRNAs that might promote M2 polarization [56]; among them, miR-124 and miR-34a were predicted to target SOCS3 and only miR-34a was predicted to target IncGBP9. More importantly, miR-34a could promote M2 macrophage polarization [40]. Thus, we hypothesize that miR-34a might participate in IncGBP9 function on macrophage polarization.

To validate the hypothesis, we examined the expression of miR-34a in vivo and in vitro, including in M0, $\mathrm{M} 1$, and $\mathrm{M} 2$ macrophages. In SCI mice, miR-34a expression was significantly downregulated on day 28 after operation (Fig.S2A); in SCl mice infected with Adv-sh-IncGBP9, miR-34a expression was significantly upregulated on day 28 after operation, compared to Adv-sh-NC group (Fig.S2B). As shown in Fig.5A, miR34a expression was dramatically upregulated in M2 macrophages. In M1 macrophages, miR-34a expression was significantly increased by IncGBP9 silencing (Fig.5B). To investigate the cellular effects of miR-34a, we conducted miR-34a overexpression in M1 macrophages by transfection of miR-34a mimics into M0 macrophages before polarization, as confirmed by real-time PCR (Fig. 5C). LncGBP9 expression was significantly downregulated by miR-34a overexpression in M1 macrophages (Fig.5D). Consistently, the SOCS3 protein level was also decreased by miR-34a overexpression in M1 macrophages (Fig.5E).

In M2 macrophages, miR-34a expression was significantly downregulated by IncGBP9 overexpression (Fig.5F). Here, we conducted miR-34a inhibition M2 macrophages by transfection of miR-34a inhibitor, as confirmed by real-time PCR (Fig.5G). In M2 macrophages, IncGBP9 expression was significantly 
upregulated by miR-34a inhibition (Fig.5H). Consistently, the SOCS3 protein level was increased by miR34a inhibition in M2 macrophages (Fig.5I). These data indicate that IncGBP9 might regulate SOCS3 through miR-34a to participate in M1/2 macrophage polarization.

\section{LncGBP9 serves as a ceRNA for miR-34a to counteract miR-34a-mediated SOCS3 suppression}

To validate the predicted targeting of miR-34a to IncGBP9 and SOCS3, we performed luciferase reporter assays by constructing wild- and mutant-type GBP9 and SOCS3 3'-UTR luciferase reporter vectors (wtGBP9/SOCS3 3'-UTR or mut-GBP9/SOCS3 3'-UTR) as described in M\&M section (Fig.6A-B). Next, 293T cells were co-transfected with the above-described vectors and miR-34a mimics/inhibitor and examined for the luciferase activity. As shown in Fig.6A-B, the luciferase activity of wt-GBP9 and wt-SOCS3 3'-UTR vectors could be significantly inhibited by miR-34a overexpression and enhanced by miR-34a inhibition; in responding to the mutation at the putative miR-34a binding sites, the changes in the luciferase activity were abolished. Moreover, in the RNA derived from precipitated AGO2 (protein argonaute-2) protein, IncGBP9 and miR-34a levels were significantly higher than those in IgG in M1 macrophages (Fig.6C). We also performed RIP assay in M1 macrophages transfected with control miRNA (NC mimics) or miR-34a mimics and then detected IncGBP9 and miR-34a levels associated with AGO2; the results shown in Fig.6D confirmed the interaction between IncGBP9 and miR-34a. Furthermore, in IncGBP9-overexpressing M1 macrophages, the level of IncGBP9 detected was dramatically higher than that of SOCS3 and $\beta$-actin (used as a negative control) (Fig.6E), indicating that IncGBP9 and SOCS3 could bind miR-34a, respectively; IncGBP9 competes with SOCS3 for miR-34a binding.

\section{LncGBP9/miR-34a axis modulates macrophage polarization via affecting the balance of STAT1/STAT6}

After confirming the binding of miR-34a to IncGBP9 and SOCS3, next, we evaluated the dynamic effects of IncGBP9 and miR-34a on STAT1/STAT6 and macrophage polarization. M0 macrophages were cotransfected with Ad-sh-IncGBP9 and miR-34a inhibitor and then polarized to M1 macrophages, the the mRNA expression and protein levels of STAT1, p-STAT1, SOCS3, iNOS, and CD16, and the production of IL-6 and IL-12 were examined. As shown in Fig.7A, C, and E, IncGBP9 silencing significantly reduced, while miR-34a inhibition significantly increased the mRNA expression and protein levels of p-STAT1, SOCS3, iNOS, and CD16/32, as well as the production of IL-6 and IL-12 in M1 macrophages; the effects of IncGBP9 silencing could be significantly reversed by miR-34a inhibition.

M0 macrophages were co-transfected with Ad-IncGBP9 and miR-34a mimics and then polarized to M2 macrophages, the mRNA expression and protein levels of STAT6, p-STAT6, SOCS3, Arg1, and CD206, and the production of IL-10 and TGF- 31 were examined. As shown in Fig.7B, D, and E, IncGBP9 overexpression significantly increased SOCS3 mRNA expression and protein level, decreased p-STAT6, Arg1, and CD206 mRNA and protein levels, and suppressed the production of IL-10 and TGF- $\beta 1$; miR-34a overexpression exerted opposing effects on these indicators; the effects of IncGBP9 overexpression could be significantly reversed by miR-34a overexpression. These data indicate that the IncGBP9/miR-34a axis modulates M1/2 macrophage polarization through SOCS3 and STAT1/STAT6. 


\section{STAT6 binds miR-34a promoter to activate its transcription}

As predicted by the online tool, STAT6 might bind the promoter region of miR-34a to activate its transcription. STAT6 overexpression or silencing was conducted in M0 macrophages by transfection of STAT6-overexpressing or si-STAT6 vector following M2 polarization, as confirmed by Immunoblotting (Fig.8A). In M2 macrophages, the expression of miR-34a was significantly upregulated by STAT6 overexpression while downregulated by STAT6 silencing (Fig.8B). Next, wild- and mutant-type miR-34a luciferase reporter vectors are constructed; mut-miR-34a vector contained a 9-bp mutation in any of the predicted STAT6 binding sites (Fig.8C). STAT6 and wt- or mut-miR-34a promoter were then co-transfected in $\mathrm{M} 0$ macrophages followed by M2 polarization; the luciferase activity was determined. As shown in Fig.8D, the promoter activity of wt-miR-34a was dramatically increased by STAT6 overexpression; however, after mutating any of the predicted binding sites, STAT6 overexpression-induced increase in promoter activity was abolished (Fig.8D). Moreover, the ChIP assay showed that the level of STAT6 antibody binding to miR-34a binding element in the miR-34a promoter was much greater than that of IgG in M2 macrophages (Fig.8E), suggesting that STAT6 might bind to the promoter of miR-34a to activate its expression in M2 macrophages.

\section{Discussion}

In the present study, we constructed the SCI model in Balb/c mice and observed the inappropriate activation of macrophage phenotypes (macrophage polarization) after $\mathrm{SCl}$. BMDMs were isolated and identified. LncRNA GBP1P1 (IncGBP9 in mice) has been previously reported to be upregulated and observed overexpressed in M1 macrophages in the present study. SOCS3 and p-STAT1, key factors in macrophage polarization, were also overexpressed in $\mathrm{M} 1$ and underexpressed in $\mathrm{M} 2$ macrophages while p-STAT6 was underexpressed in M1 and overexpressed in M2 macrophages. Consistently, IL- 6 and IL-12 were increased in M1 while IL-10 and TGF- $\beta 1$ were increased in M2 macrophages. In M1 macrophages, IncGBP9 silencing significantly decreased p-STAT1 and SOCS3 expression and protein levels, as well as the production of IL-6 and IL-12; in M2 macrophages, IncGBP9 overexpression increased SOCS3 expression and protein levels while suppressed p-STAT6 levels and the production of IL-10 and TGF- $\beta 1$, indicating that IncGBP9 overexpression promotes the M1 polarization of macrophages. In IncGBP9silenced $\mathrm{SCl}$ mice, the BMS scores were significantly lower from day 14 after operation and the M2 polarization was promoted on day 28 after operation, further indicating that IncGBP9 silencing revised the predominance of $\mathrm{M} 1$ polarization at the late stage of secondary injury after $\mathrm{SCl}$, therefore improving the repair after $\mathrm{SCl}$. In macrophages, IncGBP9 competed with SOCS3 for miR-34a binding to counteract miR-34a-mediated suppression on SOCS3, therefore modulating STAT1/STAT6 signaling and the polarization of macrophages. Finally, STAT6 bound the promoter of miR-34a to activate its transcription, therefore forming two different regulatory loops to modulate the polarization of macrophages after SCl (Fig.8F).

As mentioned earlier, during the occurrence and development of $\mathrm{SCl}$, via enhanced phagocytosis and increased production and release of pro-inflammatory cytokines, M1 macrophages promote innate 
immunity to remove foreign microorganisms and wound fragments from damaged sites. Differently, M2 macrophages have tissue repair properties, showing a decrease in inflammatory cytokines and in the production of ROS $[57,58]$. These stimuli induce M2 macrophages to regulate inflammatory reactions, remove debris, and facilitate tissue remodeling and repair. This sequential M1-M2 macrophage response will lead to successful SCl injury repair [59]. That is, inappropriate activation of macrophage phenotypes (macrophage polarization) may cause SCI repair to fail. At the early phase after the injury, the production and release of certain inflammatory cytokines would be induced by macrophages $[44,60]$. During the early stage of $\mathrm{SCl}$ in the SCI mice model, Kigerl et al. [45] reported that $\mathrm{M} 1$ macrophages accounted for the majority. After SCl, M1 and M2 biomarkers all increased rapidly; however, Arg1, one of the M2 biomarkers, was only transient and reverted to the baseline 7 days later post-SCI [45]. On the 14th day after SCl, CD206, another M2 biomarker, was significantly increased in comparison to the normal spinal cord tissue. Differently, M1 marker iNOS transient increased until day 3 after the injury; CD32, another M1 marker, significantly decreased on day 28 post-SCI. It appears that the expression of iNOS and Arg1 is regulated by each other, suggesting that not all M1 and M2 biomarkers change their expression in a coordinated manner after $\mathrm{SCl}$, possibly because the regulation of macrophage polarization begins at different time and at different phases post-SCl, or the inflammatory microenvironment affects these factors in different manners. In the present study, we observed the levels of M1 markers, including iNOS, $\mathrm{CD} 16 / 32$, and IFN-y increased after SCl from day 3 to day 28 in a time-dependent manner. On the contrary, the expression of one of the M2 markers, Arg1, reached peak values on day 7 and 14 while decreased on day 28 after SCl. Another two M2 markers, CD206 and IL-4, reached peak values on day 7 while decreased since then. These findings indicate that the predominance of M1 macrophages continues to the late phase after $\mathrm{SCl}$. The inappropriate activation of macrophage phenotypes (macrophage polarization) might contribute to the failure of SCI repair.

As we have mentioned, the polarization of macrophages could be regulated by different stimuli and factors, including protein-coding and non-coding RNAs. The genes related to the inappropriate activation of macrophage phenotypes (macrophage polarization) might, in turn, contribute to the dysfunction of macrophages. Huang et al. analyzed the differentially-expressed genes in M0, M1, and M2 macrophages and demonstrated that 2528 mRNAs were overexpressed and 4534 mRNAs were underexpressed in the M2 group compared with the M1 group. More importantly, there was a significant steady-state in the expression levels of 275 mRNAs between three groups [38]. Another group identified a total of 1,253 differentially-expressed genes between M1 and M2 macrophages, of which 696 were upregulated and 557 downregulated in M1 macrophages compared with M2 macrophages [55]. Based on these previous findings, we performed Protein-Protein Interaction analysis and revealed that TNF, SOCS3, and STAT1 were at the core location of macrophage polarization. SOCS proteins are a family of eight intracellular cytokine-inducible proteins $[61,62]$ obtaining a basal expression in cells. SOCSs could be sharply induced by many stimuli, including cytokines, TLR ligands, immune complexes, and hormones [63]. Although SOCSs are expressed at a very low level in macrophages, they could also be rapidly induced upon activation. SOCS1 and SOCS3 can regulate the polarization of macrophages to M1 and/or M2 subtypes $[64,65]$. More importantly, IFN-y/STAT1, IL-4/STAT6, and IL-12/STAT4 signaling pathways in 
differentiating Th cells may be under feedback regulation by SOCS [66-68]. Yu et al. [69] revealed that in STAT1 $1^{-/-}$Th2 cells, SOCS1 and SOCS3 protein levels are remarkably reduced; in addition, they also demonstrated that SOCS1 and SOCS3 could lead to the suppression of STAT6 signaling. In the present study, we observed that the phosphorylation of STAT1 dramatically increased in M1 macrophages while STAT6 phosphorylation increased in M2 macrophages. Consistently, SOCS3 expression was upregulated in both $\mathrm{M} 1$ and $\mathrm{M} 2$ macrophages compared to $\mathrm{M} 0$ type but was significantly higher in $\mathrm{M} 1 \mathrm{compared}$ to M2 macrophages. These findings suggest that STAT1/STAT6 signaling and SOCS3 might participate in the inappropriate activation of macrophage phenotypes (macrophage polarization). Regarding differentially-expressed IncRNAs, IncRNA GBP1P1 (IncGBP9 in mice) was significantly upregulated in M1 macrophages according to previous studies (GSE117040 and GSE5099) and our observations, suggesting that IncGBP9 may play a role in M1/M2 macrophage polarization.

As we have mentioned, GBP1P1 is a pseudogene of the guanylate-binding protein of GB (guanylatebinding protein). GBPs account for over $20 \%$ of the proteins induced after IFN-y treatment [70, 71]. GBP family is also involved in macrophage functions, such as IFN- - -mediated macrophage activation and immune defense [54]. More importantly, IncRNA GBP1P1 expression was significantly up-regulated in M1 macrophages according to several online data (Fig.S1). In the present study, by conducting IncGBP9 silencing in M1 macrophages, we observed a decreased expression of SOCS3 and suppressed phosphorylation of STAT1, as well as reduced production of IL-6 and IL-12. On the contrary, IncGBP9 overexpression in M2 macrophages significantly induced the upregulation of SOCS3 while suppressed the phosphorylation of STAT6 and the production of IL-10 and TGF- $\beta 1$. Notably, the effects of IncGBP9 silencing on M1 macrophages were significantly reversed by SOCS3 overexpression while the effects of IncGBP9 overexpression on M2 macrophages were significantly reversed by SOCS3 silencing. Consistently, in SCI mice model, IncGBP9 silencing significantly suppressed STAT1 phosphorylation and SOCS3 expression while promoted STAT6 phosphorylation on day 28 after SCl; in the meantime, IncGBP9 silencing caused a significant decrease in the BMS scores, indicating that IncGBP9 silencing inhibits SOCS3 while promotes STAT6 activation at the late phase of SCl, therefore improving the SCI repair.

It has recently been discovered that IncRNAs act as miRNA "sponges" by sharing common miRNAs responses elements (MRE) and inhibiting the targeting activity of miRNAs on downstream target mRNAs, therefore forming posttranscriptional ceRNA networks to regulate the expression of downstream target mRNAs and participating in biological processes [34]. We have revealed that IncGBP9 plays an essential role in M1/M2 macrophage polarization via SOCS3 and STAT1/STAT6, here, we hypothesize miRNAs might mediate the function of IncGBP9 in macrophage polarization. miR-34a, previously known for its potent tumor-suppressive role, has been regarded as an inflammation regulator. Jiang et al. [40] reported that the expression of miR-34a was downregulated in macrophages after LPS stimulation. MiR-34a overexpression decreased the expression of inflammatory cytokines TNF- $\alpha$ and IL- 6 in LPS-treated RAW264.7 cells. Furthermore, LPS-induced NF-KB activation was also significantly suppressed by miR34a. In the present study, online tools predicted that miR-34a might target both IncGBP9 and SOCS3. Consistent with the previous studies, miR-34a expression was significantly downregulated in M1 
macrophages after LPS + IFN-y stimulation while upregulated in M2 macrophages. miR-34a overexpression in M1 macrophages significantly inhibited IncGBP9 expression and reduced SOCS3 protein levels, on the contrary, miR-34a inhibition in M2 macrophages promoted the expression of IncGBP9 and the protein levels of SOCS3. Regarding the molecular mechanism, miR-34a directly targets IncGBP9 and SOCS3 3'-UTR. LnCGBP9 competed with SOCS3 for miR-34a binding, therefore abolishing miR-34a-mediated SOCS3 suppression. LncGBP9 silencing significantly decreased the levels of SOCS3 and M1 macrophage markers, while IncGBP9 overexpression increased SOCS3 while reduced the levels of M2 macrophage markers. In both macrophage types, the effects of miR-34a were opposite to those of IncGBP9. More importantly, the effects of IncGBP9 could be significantly reversed by miR-34a, indicating that IncGBP9 exerts its functions in macrophage polarization via serving as a ceRNA for miR-34a to counteracting miR-34a-mediated SOCS3 suppression.

Interestingly, the phosphorylation of STAT6 and miR-34a expression is significantly upregulated in M2 macrophages. Previously, STAT6 has been regarded as the dominant mediator in IL-4-induced transcriptional alterations in macrophages [72]; herein, we speculated that STAT6 might also take the responsibility for the IL-4-induced miR-34a upregulation in macrophages. As predicted by online tools and later confirmed by luciferase reporter and ChIP assays, STAT6 binds the promoter region of miR-34a to activate its transcription.

\section{Conclusions}

In summary, in macrophages, IncGBP9 competed with SOCS3 for miR-34a binding to counteract miR-34amediated suppression on SOCS3, therefore modulating STAT1/STAT6 signaling and the polarization of macrophages. STAT6 bound the promoter of miR-34a to activate its transcription, therefore forming two different regulatory loops to modulate the polarization of macrophages after $\mathrm{SCl}$ (Fig.8F). We provide a novel strategy for improving the failure in $\mathrm{SCl}$ repair.

\section{Abbreviations}

BMDMs, bone marrow-derived macrophages; ChIP, Chromatin immunoprecipitation; CNS, central nervous system; IncRNAs, long non-coding RNAs; miRNAs, microRNAs; PGE2, prostaglandin; RIP, RNA Immunoprecipitation; RNS, reactive nitrogen; ROS, reactive oxygen species; SCl, spinal cord injury; SOCS3, suppressor of cytokine signaling 3; STAT1, signal transducer and activator of transcription 1; STAT6, signal transducer and activator of transcription 6.

\section{Declarations}

Ethics approval and consent to participate: All procedures performed in studies involving animals were in accordance with the ethical standards of The Third Xiangya Hospital, Central South University and with the 1964 Helsinki declaration. Informed consent to participate in the study has been obtained from participants. 
Consent for publication: Consent for publication was obtained from the participants.

Availability of data and materials: Please contact the authors for data requests.

Conflict of Interest: The authors declare that they have no competing interests.

Funding: This work was supported by National Natural Science Foundation of China (81902224), the Natural Science Foundation of Hunan Province (Grant Nos. 2019JJ50959).

Author Contributions: Zhiyue Li, Qun Zhao made substantial contribution to the conception and design of the work; Tianding Wu, Yong Cao analyzed and interpreted the data; Zhiyue Li, Qiancheng Zhao drafted the manuscript; Jiahui Zhou revised the work critically for important intellectual content; Final approval of the work: all authors.

Acknowledgments: None.

\section{References}

1. Xu, D., et al., Expression of Nemo-like kinase after spinal cord injury in rats. J Mol Neurosci, 2014. 52(3): p. 410-8.

2. Khalatbary, A.R. and G.R. Zarrinjoei, Anti-inflammatory effect of oleuropein in experimental rat spinal cord trauma. Iran Red Crescent Med J, 2012. 14(4): p. 229-34.

3. Katoh, S., et al., High incidence of acute traumatic spinal cord injury in a rural population in Japan in 2011 and 2012: an epidemiological study. Spinal Cord, 2014. 52(4): p. 264-7.

4. Collins, W.F., A review and update of experiment and clinical studies of spinal cord injury. Paraplegia, 1983. 21(4): p. 204-19.

5. Tator, C.H., Update on the pathophysiology and pathology of acute spinal cord injury. Brain Pathol, 1995. 5(4): p. 407-13.

6. Agrawal, S.K. and M.G. Fehlings, Mechanisms of secondary injury to spinal cord axons in vitro: role of $\mathrm{Na}+\mathrm{Na}(+)-\mathrm{K}(+)$-ATPase, the $\mathrm{Na}(+)-\mathrm{H}+$ exchanger, and the $\mathrm{Na}(+)-\mathrm{Ca} 2+$ exchanger. J Neurosci, 1996. 16(2): p. 545-52.

7. Beattie, M.S., et al., Cell death in models of spinal cord injury. Prog Brain Res, 2002. 137: p. 37-47.

8. Schwartz, G. and M.G. Fehlings, Secondary injury mechanisms of spinal cord trauma: a novel therapeutic approach for the management of secondary pathophysiology with the sodium channel blocker riluzole. Prog Brain Res, 2002. 137: p. 177-90.

9. Profyris, C., et al., Degenerative and regenerative mechanisms governing spinal cord injury. Neurobiol Dis, 2004. 15(3): p. 415-36.

10. Hauben, E. and M. Schwartz, Therapeutic vaccination for spinal cord injury: helping the body to cure itself. Trends Pharmacol Sci, 2003. 24(1): p. 7-12. 
11. Jakubzick, C., et al., Minimal differentiation of classical monocytes as they survey steady-state tissues and transport antigen to lymph nodes. Immunity, 2013. 39(3): p. 599-610.

12. Shechter, R. and M. Schwartz, Harnessing monocyte-derived macrophages to control central nervous system pathologies: no longer 'if' but 'how'. J Pathol, 2013. 229(2): p. 332-46.

13. Hines, D.J., et al., Microglia processes block the spread of damage in the brain and require functional chloride channels. Glia, 2009. 57(15): p. 1610-8.

14. Greenhalgh, A.D. and S. David, Differences in the phagocytic response of microglia and peripheral macrophages after spinal cord injury and its effects on cell death. J Neurosci, 2014. 34(18): p. 631622.

15. Gehrmann, J., Microglia: a sensor to threats in the nervous system? Res Virol, 1996. 147(2-3): p. 7988.

16. Paludan, S.R., Interleukin-4 and interferon-gamma: the quintessence of a mutual antagonistic relationship. Scand J Immunol, 1998. 48(5): p. 459-68.

17. Standiford, T.J., et al., IL-4 inhibits the expression of IL-8 from stimulated human monocytes. J Immunol, 1990. 145(5): p. 1435-9.

18. Wynn, T.A., IL-13 effector functions. Annu Rev Immunol, 2003. 21: p. 425-56.

19. Wynn, T.A., Fibrotic disease and the $T(H) 1 / T(H) 2$ paradigm. Nat Rev Immunol, 2004. 4(8): p. 583-94.

20. Gordon, S. and P.R. Taylor, Monocyte and macrophage heterogeneity. Nat Rev Immunol, 2005. 5(12): p. 953-64.

21. Kacimi, R., R.G. Giffard, and M.A. Yenari, Endotoxin-activated microglia injure brain derived endothelial cells via NF-kappaB, JAK-STAT and JNK stress kinase pathways. J Inflamm (Lond), 2011. 8: p. 7.

22. Jeong, J.W., et al., Anti-inflammatory effects of cordycepin via suppression of inflammatory mediators in BV2 microglial cells. Int Immunopharmacol, 2010. 10(12): p. 1580-6.

23. Block, M.L., L. Zecca, and J.S. Hong, Microglia-mediated neurotoxicity: uncovering the molecular mechanisms. Nat Rev Neurosci, 2007. 8(1): p. 57-69.

24. Kong, X. and J. Gao, Macrophage polarization: a key event in the secondary phase of acute spinal cord injury. J Cell Mol Med, 2017. 21(5): p. 941-954.

25. Arranz, A., et al., Akt1 and Akt2 protein kinases differentially contribute to macrophage polarization. Proc Natl Acad Sci U S A, 2012. 109(24): p. 9517-22.

26. Raes, G., et al., FIZZ1 and Ym as tools to discriminate between differentially activated macrophages. Dev Immunol, 2002. 9(3): p. 151-9.

27. Martinez, F.O., et al., Macrophage activation and polarization. Front Biosci, 2008. 13: p. 453-61.

28. Khan, Z., et al., Overexpression of the C-domain of angiotensin-converting enzyme reduces melanoma growth by stimulating M1 macrophage polarization. J Biol Chem, 2019.

29. Yao, A., et al., Programmed death 1 deficiency induces the polarization of macrophages/microglia to the M1 phenotype after spinal cord injury in mice. Neurotherapeutics, 2014. 11(3): p. 636-50. 
30. Nagano, T. and P. Fraser, No-nonsense functions for long noncoding RNAs. Cell, 2011. 145(2): p. 17881.

31. Batista, P.J. and H.Y. Chang, Long noncoding RNAs: cellular address codes in development and disease. Cell, 2013. 152(6): p. 1298-307.

32. Salmanidis, M., et al., Direct transcriptional regulation by nuclear microRNAs. Int J Biochem Cell Biol, 2014. 54: p. 304-11.

33. Shi, X., et al., Long non-coding RNAs: a new frontier in the study of human diseases. Cancer Lett, 2013. 339(2): p. 159-66.

34. Sen, R., et al., Competing endogenous RNA: the key to posttranscriptional regulation. ScientificWorldJournal, 2014. 2014: p. 896206.

35. Tan, J.Y. and A.C. Marques, The miRNA-mediated cross-talk between transcripts provides a novel layer of posttranscriptional regulation. Adv Genet, 2014. 85: p. 149-99.

36. Heward, J.A. and M.A. Lindsay, Long non-coding RNAs in the regulation of the immune response. Trends Immunol, 2014. 35(9): p. 408-19.

37. Recalcati, S., et al., Differential regulation of iron homeostasis during human macrophage polarized activation. Eur J Immunol, 2010. 40(3): p. 824-35.

38. Huang, Z., et al., Identification of Differentially Expressed Long Non-coding RNAs in Polarized Macrophages. Sci Rep, 2016. 6: p. 19705.

39. Reddy, M.A., et al., Regulation of inflammatory phenotype in macrophages by a diabetes-induced long noncoding RNA. Diabetes, 2014. 63(12): p. 4249-61.

40. Jiang, P., et al., MiR-34a inhibits lipopolysaccharide-induced inflammatory response through targeting Notch1 in murine macrophages. Exp Cell Res, 2012. 318(10): p. 1175-84.

41. Jakeman, L.B., et al., Traumatic spinal cord injury produced by controlled contusion in mouse. J Neurotrauma, 2000. 17(4): p. 299-319.

42. Basso, D.M., et al., Basso Mouse Scale for locomotion detects differences in recovery after spinal cord injury in five common mouse strains. J Neurotrauma, 2006. 23(5): p. 635-59.

43. Zhu, Y., et al., Macrophage Transcriptional Profile Identifies Lipid Catabolic Pathways That Can Be Therapeutically Targeted after Spinal Cord Injury. J Neurosci, 2017. 37(9): p. 2362-2376.

44. Longbrake, E.E., et al., Characterization and modeling of monocyte-derived macrophages after spinal cord injury. J Neurochem, 2007. 102(4): p. 1083-94.

45. Kigerl, K.A., et al., Identification of two distinct macrophage subsets with divergent effects causing either neurotoxicity or regeneration in the injured mouse spinal cord. J Neurosci, 2009. 29(43): p. 13435-44.

46. Burgess, A.W., et al., Purification of two forms of colony-stimulating factor from mouse L-cellconditioned medium. J Biol Chem, 1985. 260(29): p. 16004-11.

47. Wang, Q., et al., Abrogation of hepatic ATP-citrate lyase protects against fatty liver and ameliorates hyperglycemia in leptin receptor-deficient mice. Hepatology, 2009. 49(4): p. 1166-75. 
48. Shao, M., et al., Hepatic IRE1alpha regulates fasting-induced metabolic adaptive programs through the XBP1s-PPARalpha axis signalling. Nat Commun, 2014. 5: p. 3528.

49. Wang, J., et al., MiR-92b targets p57kip2 to modulate the resistance of hepatocellular carcinoma (HCC) to ionizing radiation (IR) -based radiotherapy. Biomed Pharmacother, 2019. 110: p. 646-655.

50. Li, Q., B. Yu, and P. Yang, Hypoxia-induced HMGB1 in would tissues promotes the osteoblast cell proliferation via activating ERK/JNK signaling. Int J Clin Exp Med, 2015. 8(9): p. 15087-97.

51. Li, L., et al., The role of IncRNA XIST/miR-211 axis in modulating the proliferation and apoptosis of osteoarthritis chondrocytes through CXCR4 and MAPK signaling. Biochem Biophys Res Commun, 2018. 503(4): p. 2555-2562.

52. Liu, K., et al., Wild-type and mutant $p 53$ differentially modulate miR-124/iASPP feedback following pohotodynamic therapy in human colon cancer cell line. Cell Death Dis, 2017. 8(10): p. e3096.

53. Li, X., et al., Regulation of Macrophage Activation and Polarization by HCC-Derived Exosomal IncRNA TUC339. Int J Mol Sci, 2018. 19(10).

54. Selleck, E.M., et al., Guanylate-binding protein 1 (Gbp1) contributes to cell-autonomous immunity against Toxoplasma gondii. PLoS Pathog, 2013. 9(4): p. e1003320.

55. Jiang, L., et al., Microarray and bioinformatics analyses of gene expression profiles in BALB/C murine macrophage polarization. Mol Med Rep, 2017. 16(5): p. 7382-7390.

56. Essandoh, K., et al., MiRNA-Mediated Macrophage Polarization and its Potential Role in the Regulation of Inflammatory Response. Shock, 2016. 46(2): p. 122-31.

57. Mosser, D.M. and J.P. Edwards, Exploring the full spectrum of macrophage activation. Nat Rev Immunol, 2008. 8(12): p. 958-69.

58. Galli, S.J., N. Borregaard, and T.A. Wynn, Phenotypic and functional plasticity of cells of innate immunity: macrophages, mast cells and neutrophils. Nat Immunol, 2011. 12(11): p. 1035-44.

59. Gensel, J.C. and B. Zhang, Macrophage activation and its role in repair and pathology after spinal cord injury. Brain Res, 2015. 1619: p. 1-11.

60. Pineau, I. and S. Lacroix, Proinflammatory cytokine synthesis in the injured mouse spinal cord: multiphasic expression pattern and identification of the cell types involved. J Comp Neurol, 2007. 500(2): p. 267-85.

61. Linossi, E.M., et al., Suppression of cytokine signaling: the SOCS perspective. Cytokine Growth Factor Rev, 2013. 24(3): p. 241-8.

62. Trengove, M.C. and A.C. Ward, SOCS proteins in development and disease. Am J Clin Exp Immunol, 2013. 2(1): p. 1-29.

63. Yoshimura, A., T. Naka, and M. Kubo, SOCS proteins, cytokine signalling and immune regulation. Nat Rev Immunol, 2007. 7(6): p. 454-65.

64. Qin, H., et al., SOCS3 deficiency promotes M1 macrophage polarization and inflammation. J Immunol, 2012. 189(7): p. 3439-48. 
65. Liu, Y., et al., Unique expression of suppressor of cytokine signaling 3 is essential for classical macrophage activation in rodents in vitro and in vivo. J Immunol, 2008. 180(9): p. 6270-8.

66. Fujimoto, M., et al., A regulatory role for suppressor of cytokine signaling- 1 in $T(h)$ polarization in vivo. Int Immunol, 2002. 14(11): p. 1343-50.

67. Eyles, J.L., et al., Negative regulation of interleukin-12 signaling by suppressor of cytokine signaling1. J Biol Chem, 2002. 277(46): p. 43735-40.

68. Cornish, A.L., et al., Suppressor of cytokine signaling-1 regulates signaling in response to interleukin-2 and other gamma c-dependent cytokines in peripheral T cells. J Biol Chem, 2003. 278(25): p. 2275561.

69. Yu, C.R., et al., Cell proliferation and STAT6 pathways are negatively regulated in T cells by STAT1 and suppressors of cytokine signaling. J Immunol, 2004. 173(2): p. 737-46.

70. MacMicking, J.D., Interferon-inducible effector mechanisms in cell-autonomous immunity. Nat Rev Immunol, 2012. 12(5): p. 367-82.

71. Kim, B.H., et al., IFN-inducible GTPases in host cell defense. Cell Host Microbe, 2012. 12(4): p. 43244.

72. Gordon, S. and F.O. Martinez, Alternative activation of macrophages: mechanism and functions. Immunity, 2010. 32(5): p. 593-604.

\section{Supplementary Figure Information}

Fig.S1 RNA-seq analyses GSE5099, GSE117040, E-GEOD-57494, E-MTAB-2399 and GSE40885 (A) A schematic diagram showing the process of selecting IncRNAs upregulated in M1 macrophages based on GSE117040 and GSE5099. LncRNA GBP1P1 (GBP9 in mice) and LINC00869 (Fam91a1 in mice) were selected after cross-check and literature review. (B) GSE5099 reported differentially-expressed genes at different time point during inducing the monocytes into macrophages. LncRNA GBP1P1 is significantly up-regulated in M1 macrophages compared to that in monocytes and M2 macrophages; (C) GSE117040 performed RNA-Seq analysis on RNA expression in M1 and M2 polarized human macrophages (4 replicate samples) and showed that IncRNA GBP1P1 expression was significantly up-regulated in M1 macrophages; (D) E-GEOD-57494 performed RNA-Seq analysis on RNA expression in human monocytes (cd14+/cd16+) treated with LPS + IFN- $y$, compared to DMSO treatment group. LncRNA GBP1P1 was significantly upregulated in LPS + IFN-y group 6 hours or 24 hours after the LPS + IFN-y treatment; (E) EMTAB-2399 performed RNA-Seq analysis on RNA expression in human monocytes subjected to $10 \mathrm{ng} / \mathrm{ml}$ LPS treatment. LncRNA GBP1P1 was rapidly upregulated by LPS treatment; (F) GSE40885 performed RNA-Seq analysis on RNA expression in human alveolar macrophages induced by LPS. LncRNA GBP1P1 was rapidly upregulated by LPS treatment.

Fig.S2 In vivo expression level of miR-34a in SCI mice (A) or Adv-sh-IncGBP9 infected SCI mice at day 28 of $\mathrm{SCl}$ treatment $(B)$. Values are mean \pm S.D of $n=5$ independent experiments. 


\section{Figures}
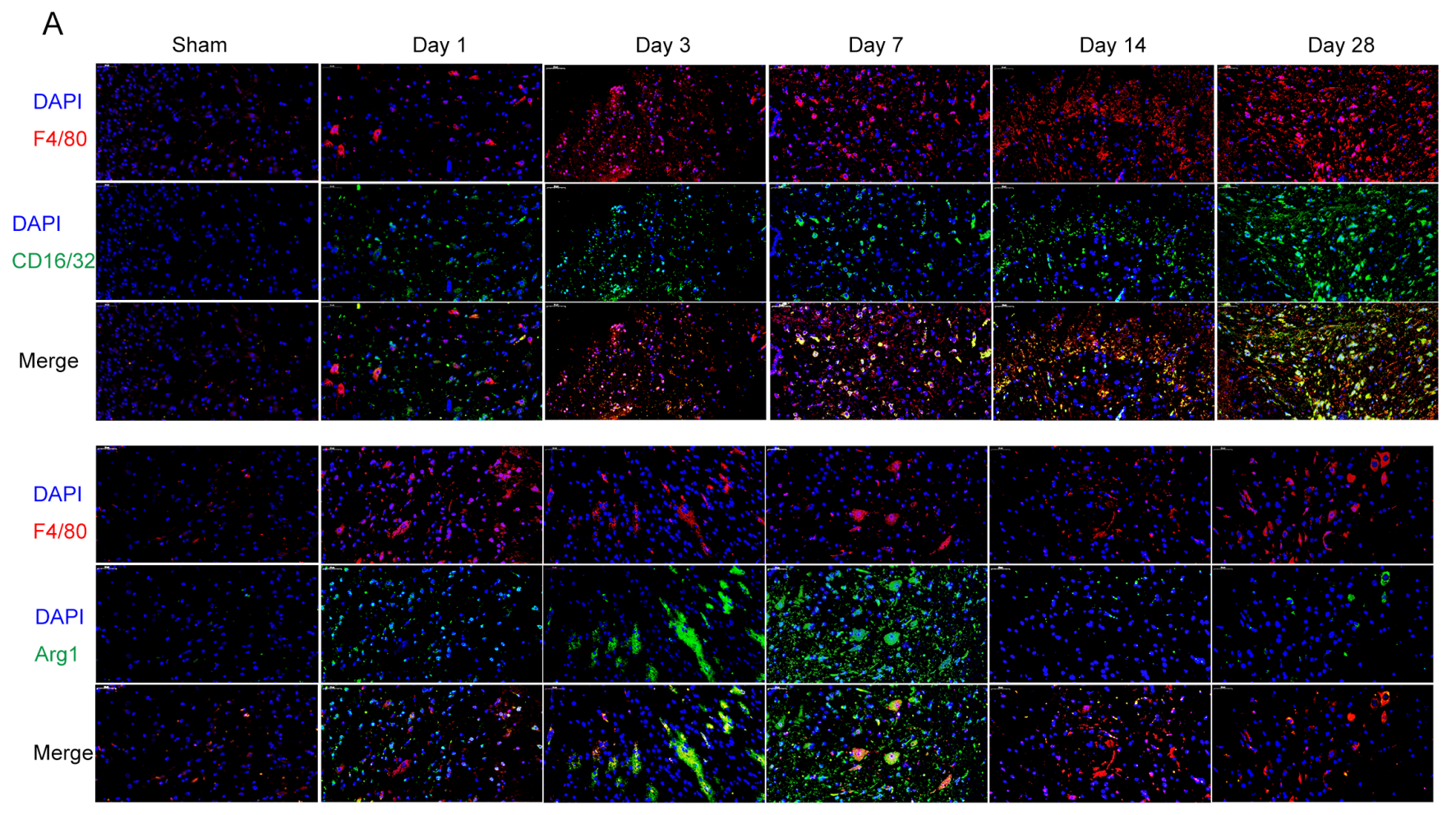

$\mathrm{B}$
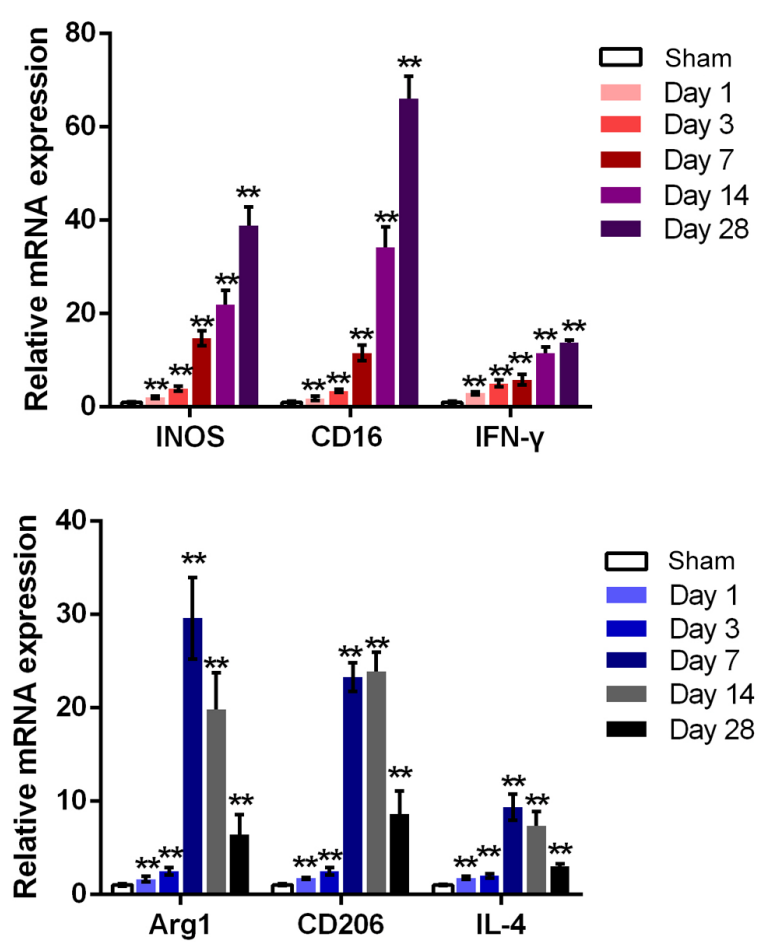

C
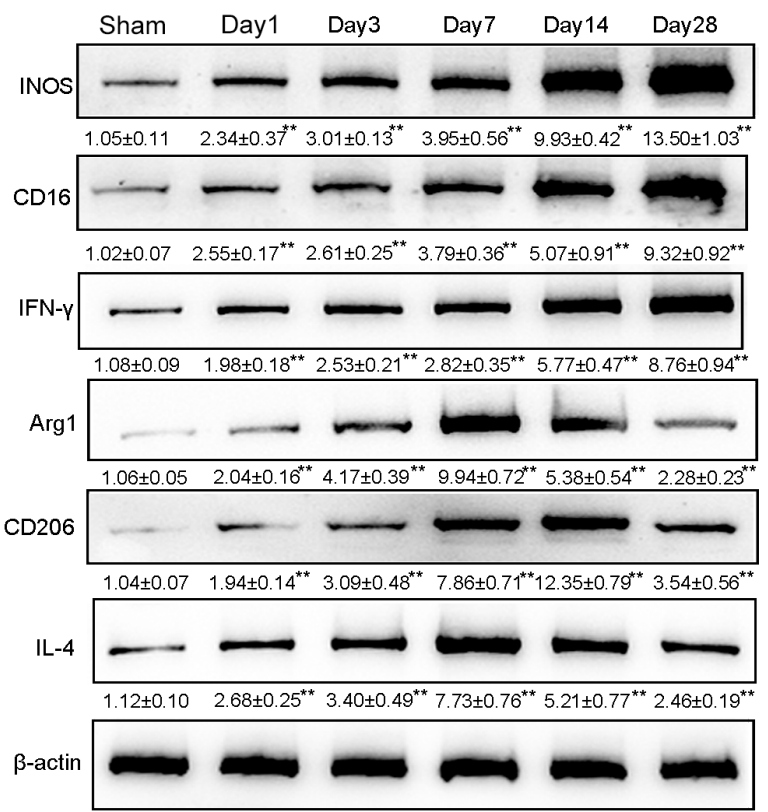

Figure 1

Macrophage polarization during spinal cord injury (SCl) (A) SCl model was constructed in Balb/c mice as described in M\&M section. $n=5$ in each group. The content and distribution of M1 macrophage marker CD16/32 and M2 macrophage marker Arg1 in spinal cords of sham mice and SCI mice were examined by 
Immunofluorescence (IF) staining on day 1, 3, 7, 14, 28 after treatment. (B) The mRNA expression and (C) the protein levels of M1 macrophage markers CD16, iNOS, and IFN- $y$ and M2 macrophage markers Arg1, CD206, and IL-4 were examined by real-time PCR $(n=5)$ and Immunoblotting on sham group or SCl group day $1,3,7,14,28$ after treatment. $n=3 * P<0.05, * * P<0.01$.

A
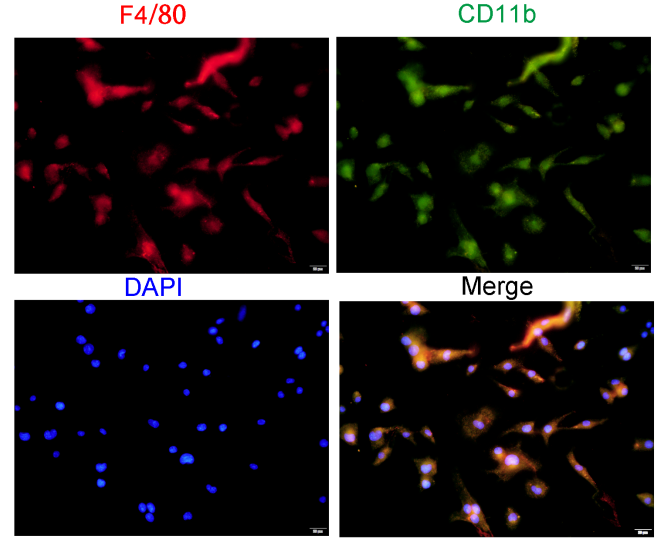

B

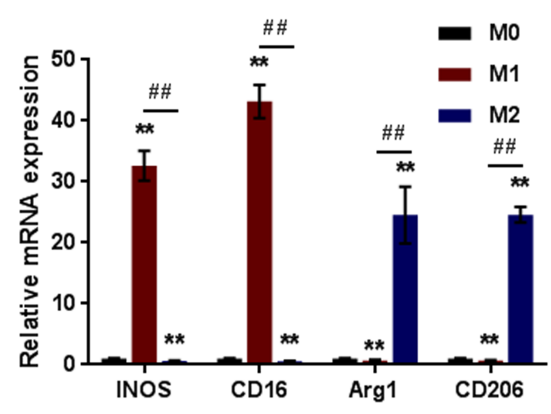

C

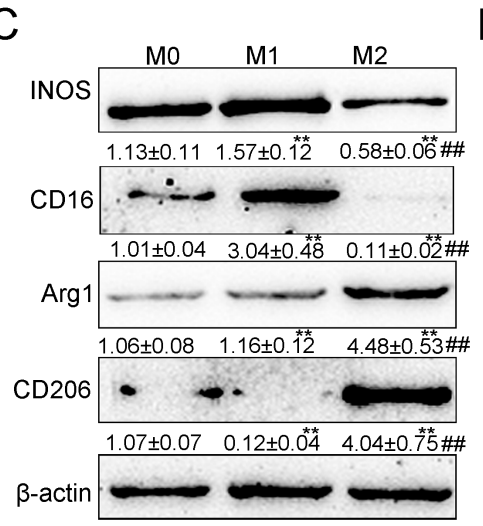

$\mathrm{F}$

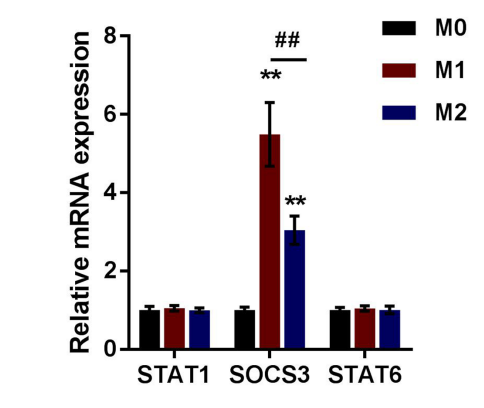

D

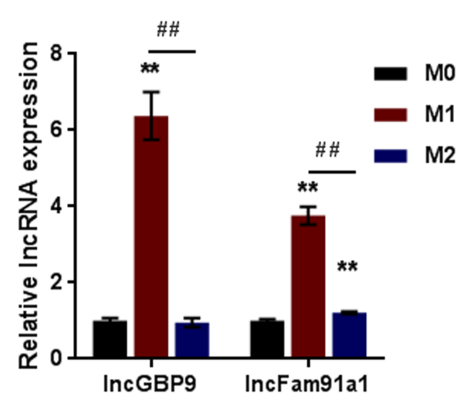

G
E

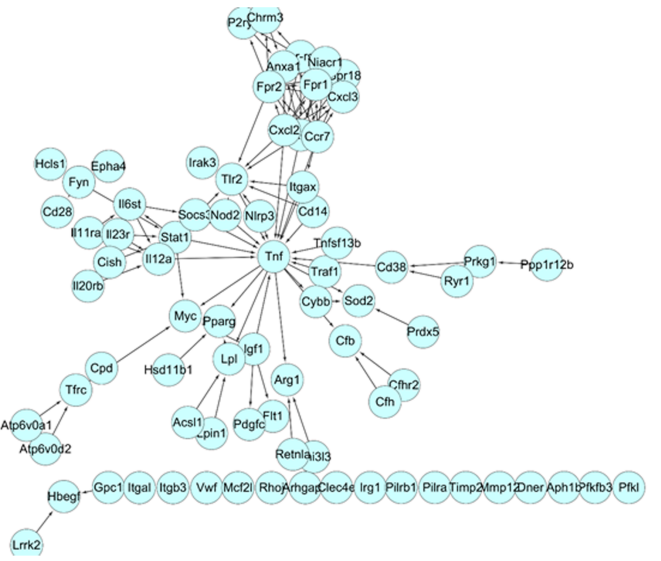

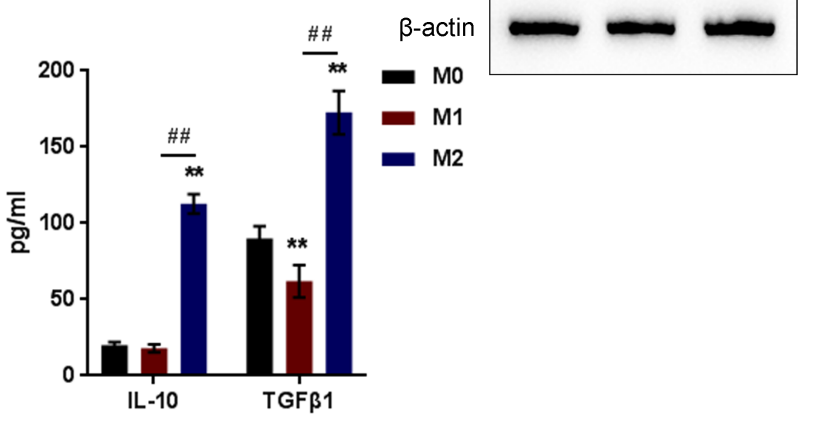
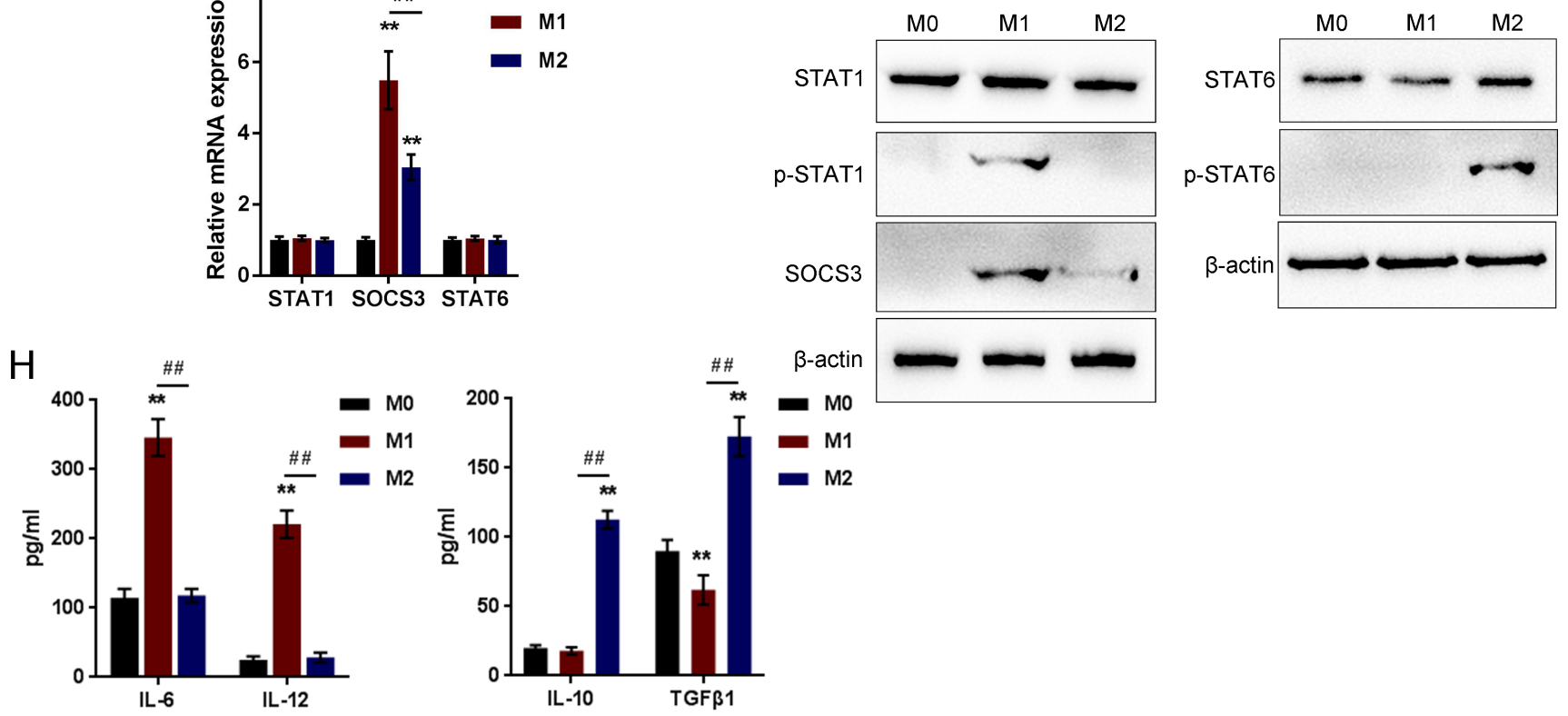

Figure 2 
Selection of IncRNAs and genes related to macrophage polarization (A) Mouse bone marrow-derived macrophages (BMDMs) were isolated and identified by examining macrophage markers $\mathrm{F} 4 / 80$ and CD11b using IF staining. (B) BMDMs were induced for differentiating towards M1 or M2 macrophages; the mRNA expression of M1 macrophage markers iNOS and CD16 and M2 macrophage markers Arg1 and CD206 were examined in M0, M1, and M2 macrophages by real-time PCR $(n=5) ;(C)$ the protein levels of M1 macrophage markers iNOS and CD16 and M2 macrophage markers Arg1 and CD206 were examined by Immunoblotting ( $n=3)$; (D) the expression of GBP9 and Fam91a1 were examined by realtime PCR. ( $n=5)$. (E) STRING analyses on differentially-expressed genes reported previously. Tnf, Socs3, and Stat1 are key factors in macrophage polarization. (F-G) The mRNA expression and protein levels of STAT1, p-STAT1, SOCS3, STAT6, and p-STAT6 in M0, M1, and M2 macrophages determined by real-time PCR $(n=5)$ and Immunoblotting $(n=3)$. (H) The production of cytokines, including IL-6, IL-12, IL-10, and TGF- $\beta 1$ in M0, M1, and M2 macrophages determined by ELISA $(n=3) .{ }^{*}<<0.05$, ${ }^{*} P<0.01$, compared to control group; $\# \mathrm{P}<0.05$, \#\#P<0.01, compared to $\mathrm{M} 1$ group. 
C
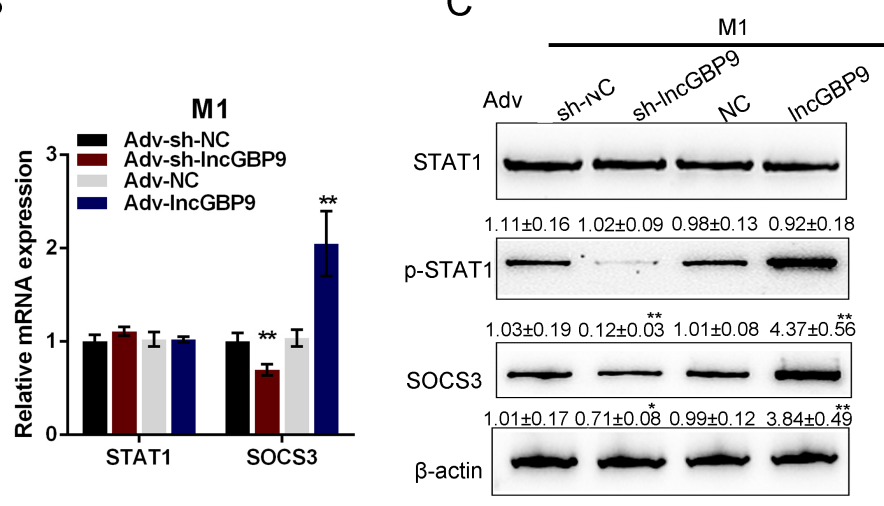

F

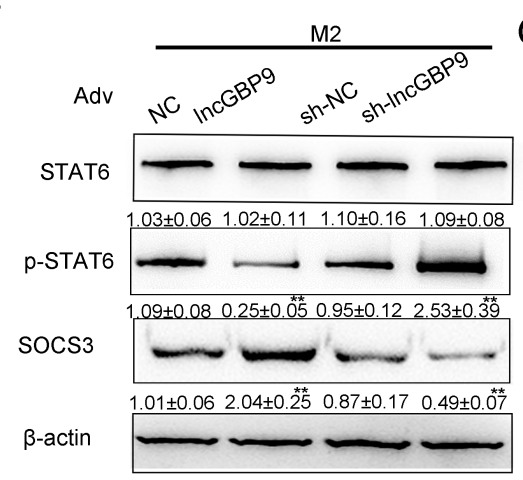

D

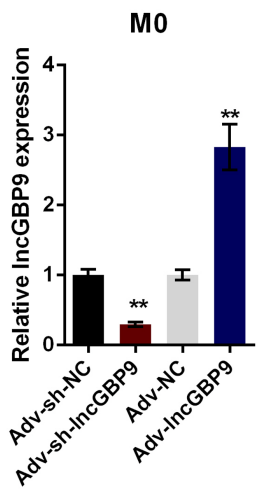

E

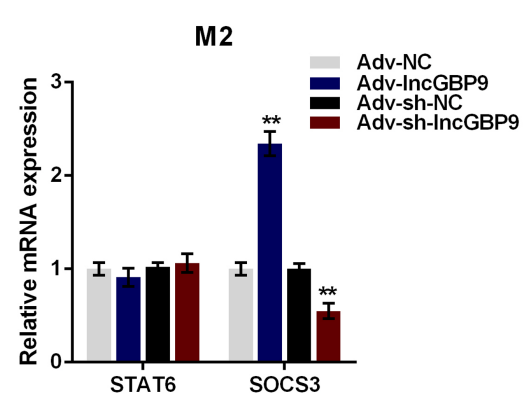

$\mathrm{H}$

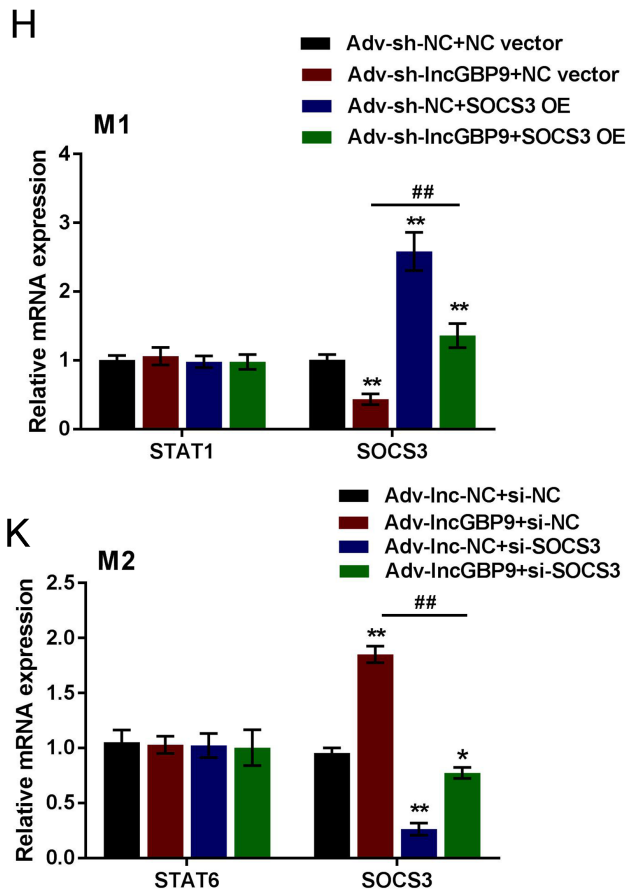

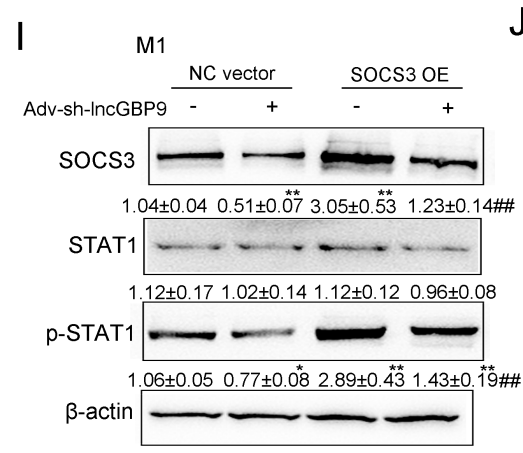

J

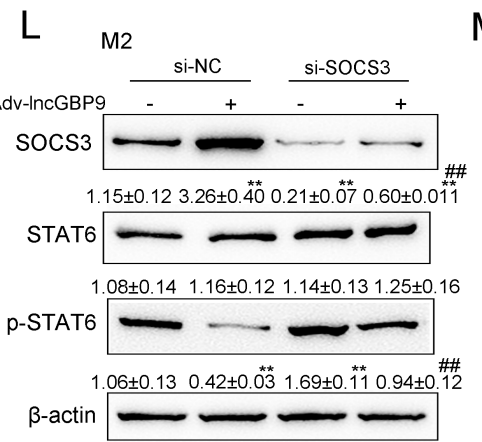

M

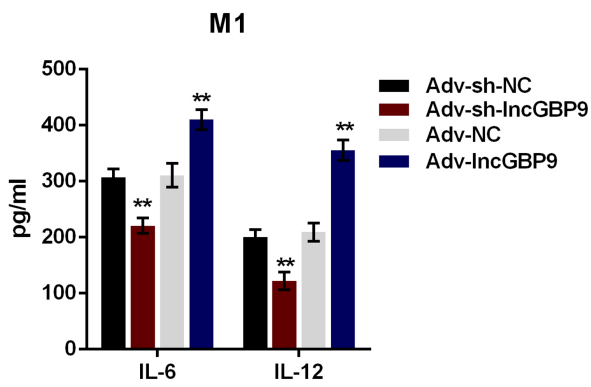

G
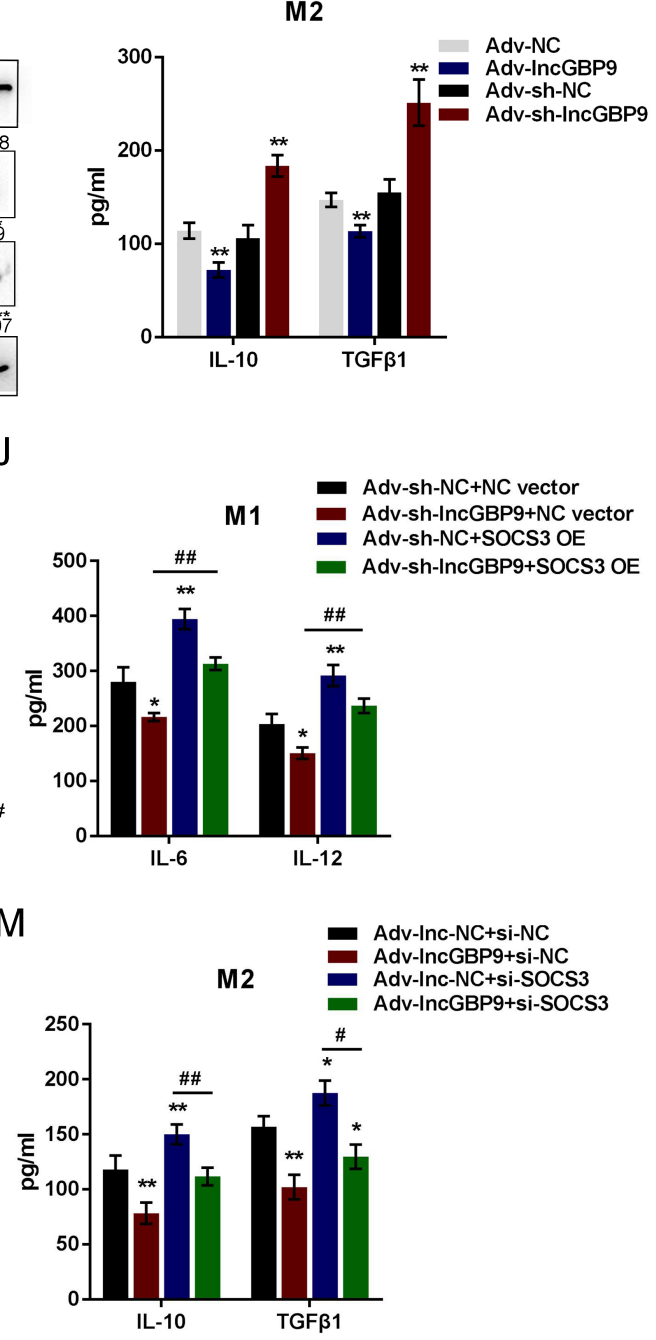

Figure 3

Effects of IncGBP9 on macrophage polarization in vitro (A) BMDMs were induced toward M0 macrophages for 7 days as described in the $M \& M$ section. Then, the silencing and overexpression of IncGBP9 conducted in M0 macrophages for 48 h, as confirmed by real-time PCR. (B-C) Adv-sh-IncGBP9 or Adv-IncGBP9 transduced M0 macrophages were induced toward M1 polarization for $48 \mathrm{~h}$ and the mRNA expression and protein levels of STAT1, p-STAT1, and SOCS3 in response to IncGBP9 silencing or 
IncGBP9 overexpression were determined by real-time PCR and Immunoblotting in M1 macrophages. (D) The production of IL- 6 and IL-12 in response to IncGBP9 silencing or IncGBP9 overexpression was determined by ELISA in M1 macrophages. (E-F) Adv-IncGBP9 or Adv-sh-IncGBP9 transduced M0 macrophages were induced toward $\mathrm{M} 2$ polarization for $48 \mathrm{~h}$ and the mRNA expression and protein levels of STAT6, p-STAT6, and SOCS3 in response to IncGBP9 overexpression or IncGBP9 silencing were determined by real-time PCR and Immunoblotting in M2 macrophages. (G) The production of IL-10 and TGF- $\beta 1$ in response to IncGBP9 overexpression or IncGBP9 silencing was determined by ELISA in M2 macrophages. Next, M1 macrophages were co-transfected with Adv-IncGBP9 and SocS3-overexpressing vector (SOCS3 OE) and examined for $(\mathrm{H})$ the mRNA of STAT1 and SOCS3 by real-time PCR; (I) the protein levels of SOCS3, STAT1, and p-STAT1 by Immunoblotting; (J) the concentrations of IL-6 and IL-12 by ELISA. M2 macrophages were co-transfected with Adv-IncGBP9 and si-SOCS3 and examined for (K) the mRNA of STAT6 and SOCS3 by real-time PCR; (L) the protein levels of SOCS3, STAT6, and p-STAT6 by Immunoblotting; (M) the concentrations of IL-10 and TGF $\beta 1$ by ELISA. ${ }^{*} P<0.05, * * P<0.01$, compared to the control group; \#P<0.05, \#\#P<0.01, compared to the Adv-IncGBP9 + NC (negative control) vector or Adv-IncGBP9 + si-NC (negative control) group. Values are mean \pm S.D of $n=3$ independent experiments.
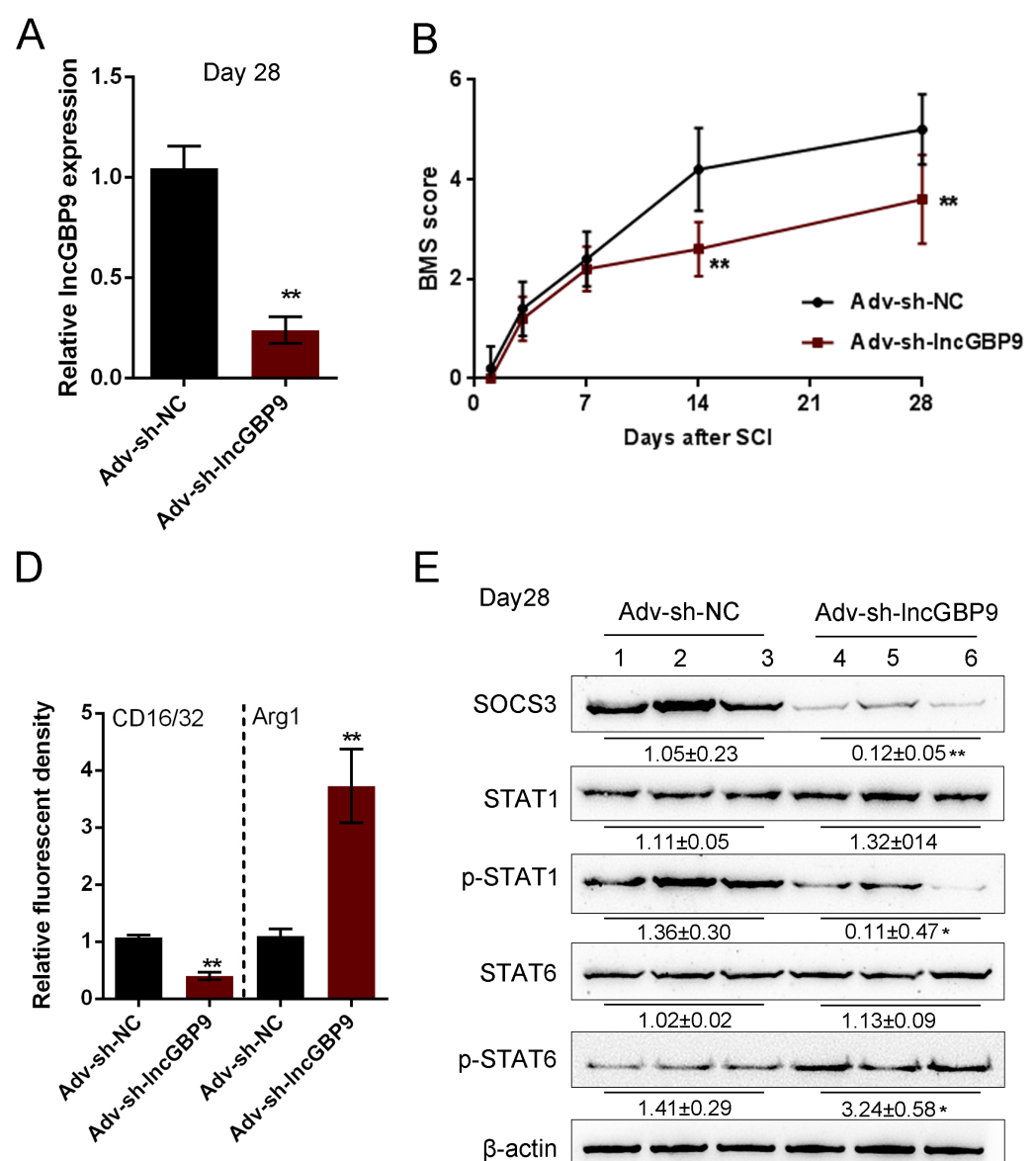

C Day28

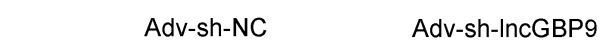

E
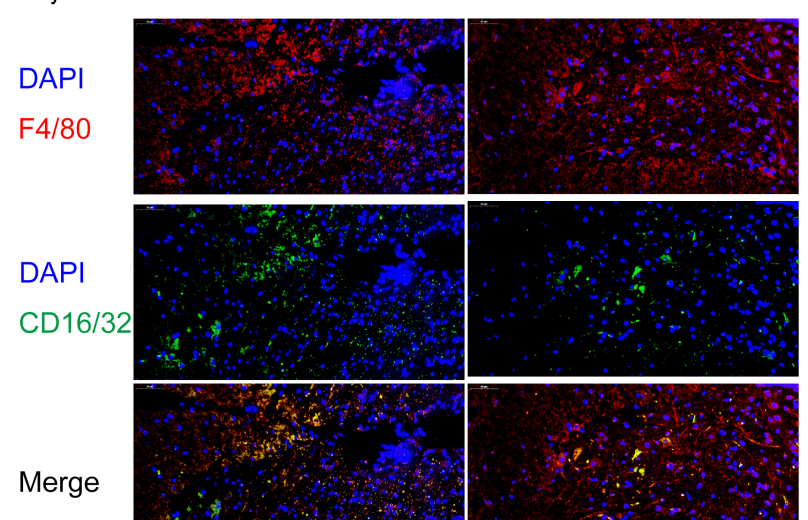

E
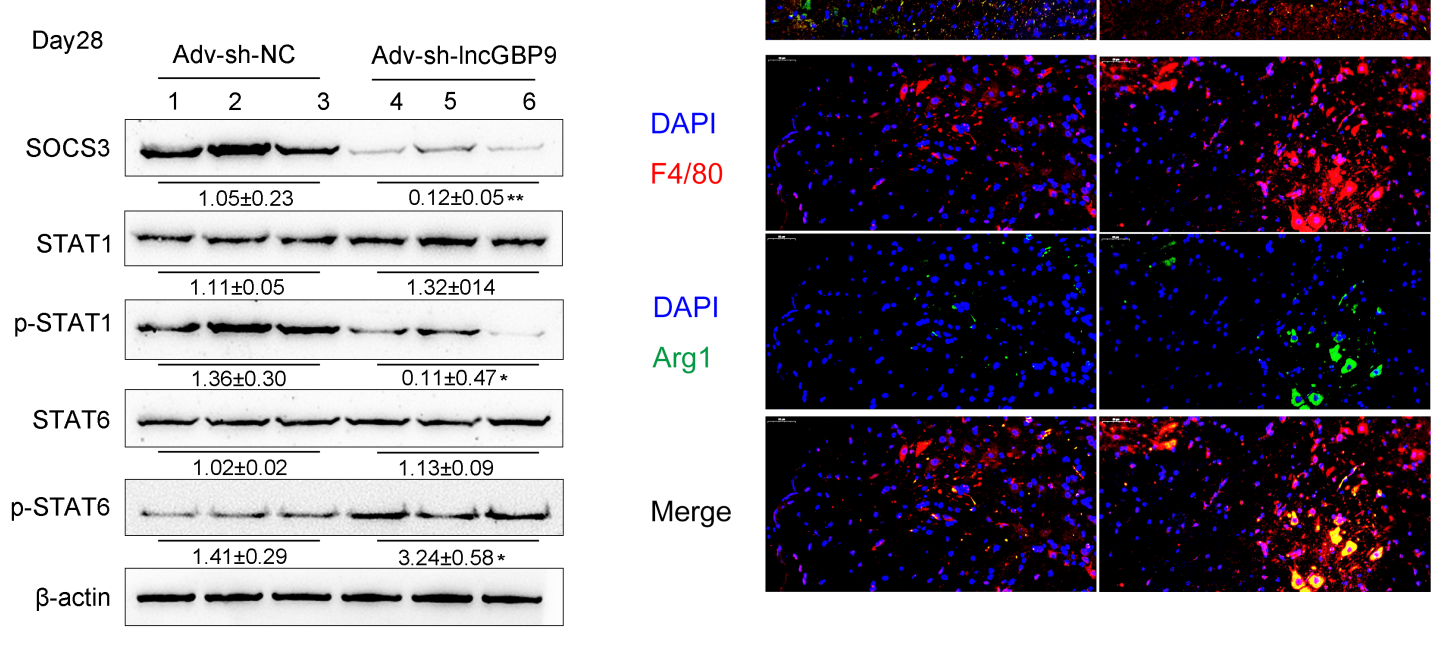

\section{Figure 4}

Effects of IncGBP9 on macrophage polarization in vivo LncGBP9 silencing was conducted in the SCI mice model via tail vein injection Ad-sh-IncGBP9. $n=5$ in each group. (A) The in vivo infection efficiency 
was verified by real-time PCR. (B) The BMS scores were evaluated on day 1, 3, 7, 14, and 28 after operation ( $n=5)$; (C-D) The content and distribution of M1 macrophage marker CD16/32 and M2 macrophage marker Arg1 determined by IF staining on day 28 after operation( $n=5)$; $(E)$ The protein levels of STAT1, p-STAT1, STAT6, p-STAT6, and SOCS3 determined by Immunoblotting on day 28 after operation $(n=3) . .{ }^{* *} P<0.01$.

A
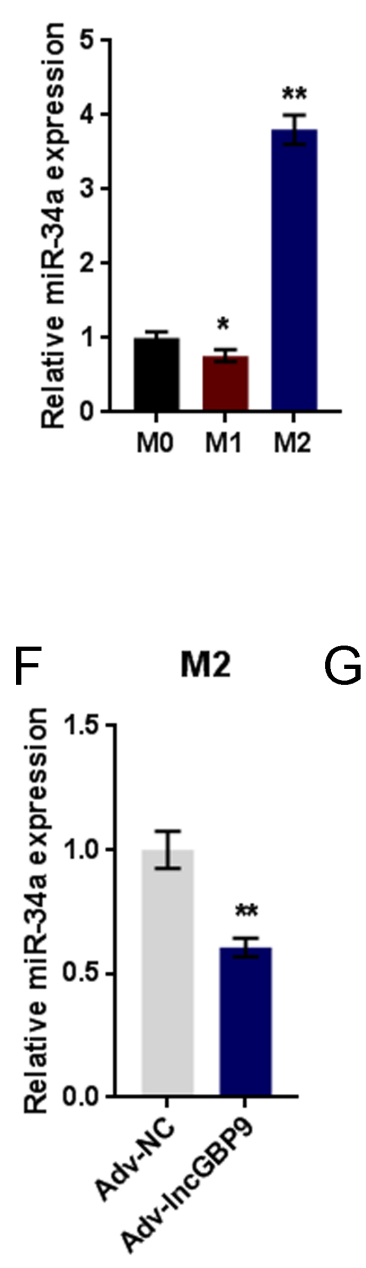

B

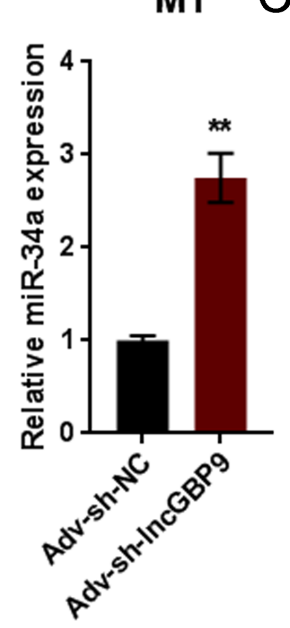

M1 D
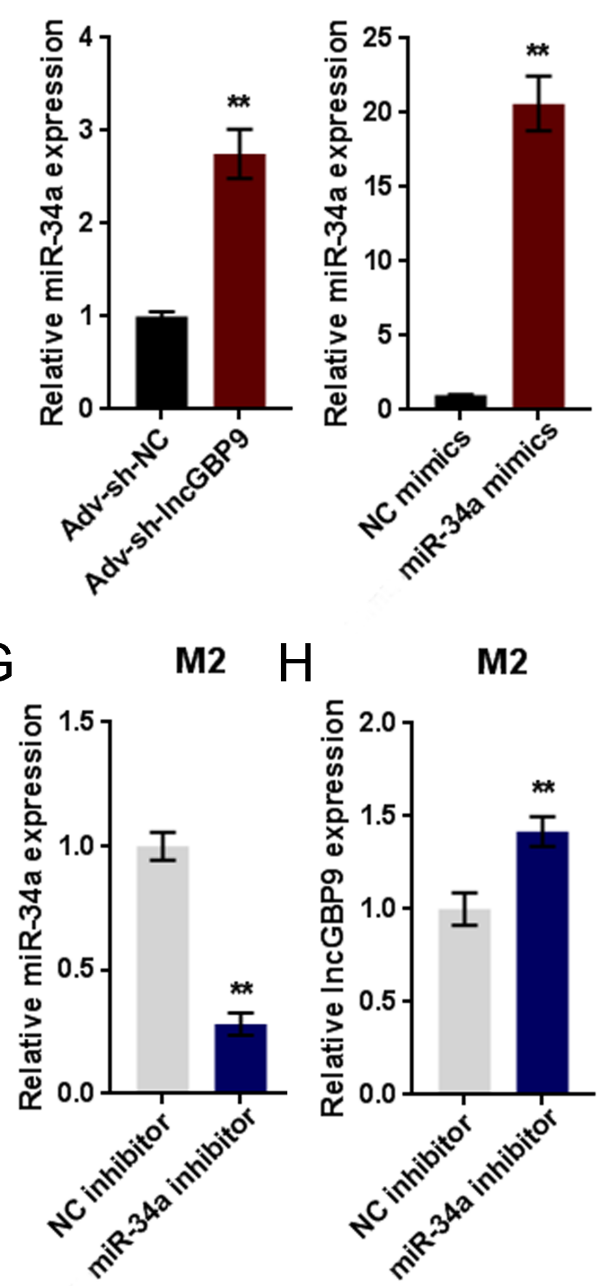

M1

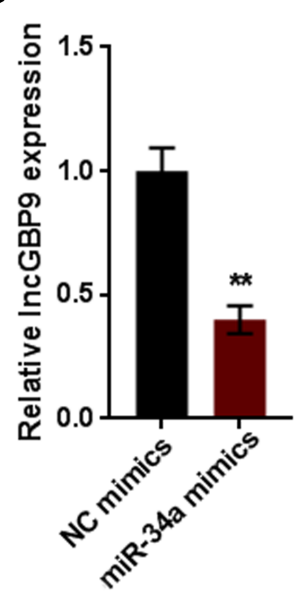

M1

$E$
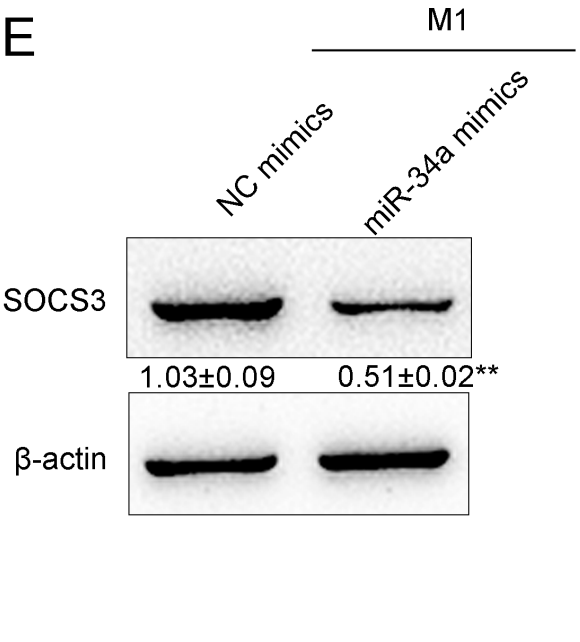

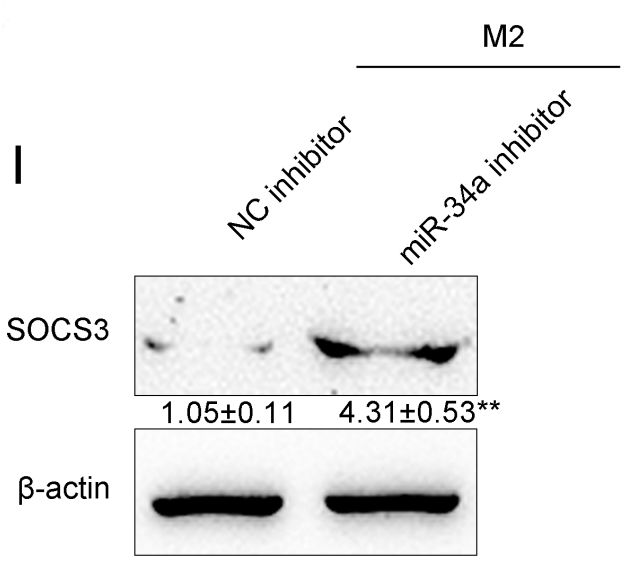

M2

socs3

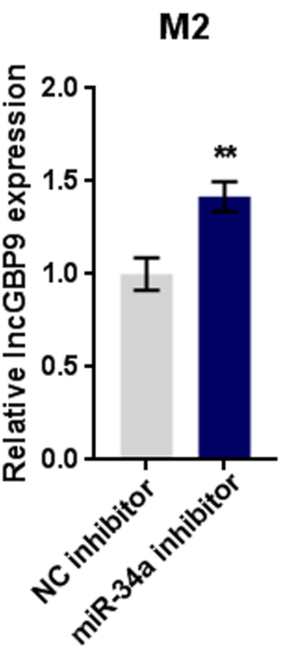

I socs3

\section{Figure 5}

LncGBP9 modulates SOCS3 through miR-34a in macrophages (A) The expression of miR-34a determined in M0, M1, and M2 macrophages by real-time PCR. (B) The expression of miR-34a in response to IncGBP9 silencing determined in M1 macrophages by real-time PCR. (C) miR-34a overexpression conducted in M1 macrophages by transfection of miR-34a mimics, as confirmed by real-time PCR. (D) LncGBP9 expression in response to miR-34a overexpression determined in M1 macrophages by real-time PCR. (E) SOCS3 protein level in response to miR-34a overexpression determined in M1 macrophages by Immunoblotting. (F) miR-34a expression in response to IncGBP9 overexpression determined in M2 macrophages by real-time PCR. (G) miR-34a inhibition conducted in M2 macrophages by transfection of 
miR-34a inhibitor, as confirmed by real-time PCR. (H) LncGBP9 expression in response to miR-34a inhibition determined in M2 macrophages by real-time PCR. (I) SOCS3 protein level in response to miR34a inhibition determined in M2 macrophages by Immunoblotting. ${ }^{*} \mathrm{P}<0.05,{ }^{*} \mathrm{P}<0.01$. Values are mean \pm S.D of $n=4$ independent experiments.

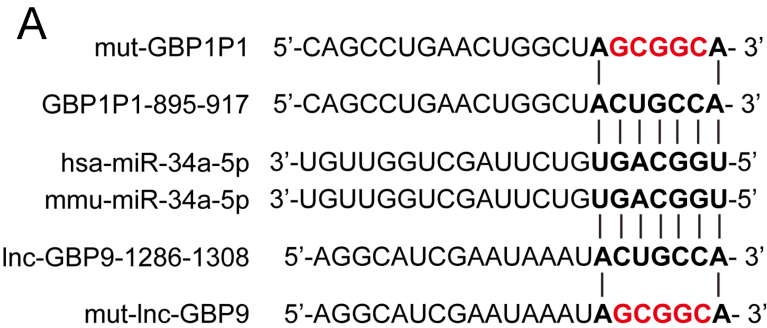

293T
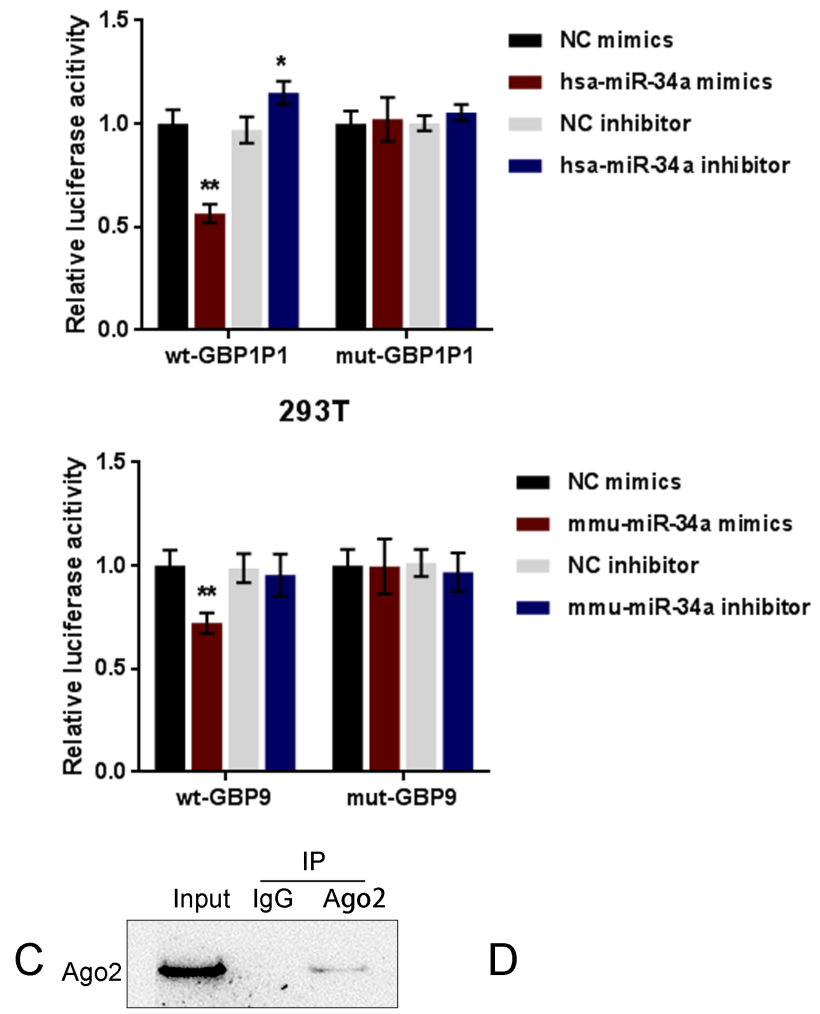

M1

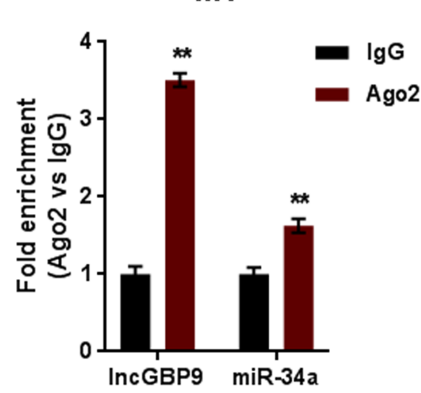

B

SOCS3 3'UTR length:1641

mut-SOCS3 5'-GCUGAACUAAUGAGAAGCGCGAG-3' SOCS3-344-350 5'-GCUGAACUAAUGAGAACUGCCAG-3' hsa-miR-34a-5p 3'-UGUUGGUCGAUUCUGUGACGGU-5 mmu-miR-34a-5p 3'-UGUUGGUCGAUUCUGUGACGGU-5' SOCS3-493-499 5'-AGAAAUAACCACUCCCACUGCCC-3' mut-SOCS3 5'-AGAAAUAACCACUCCCGCGCGGC-3' Mouse SOCS3 3'UTR length:1455

293T

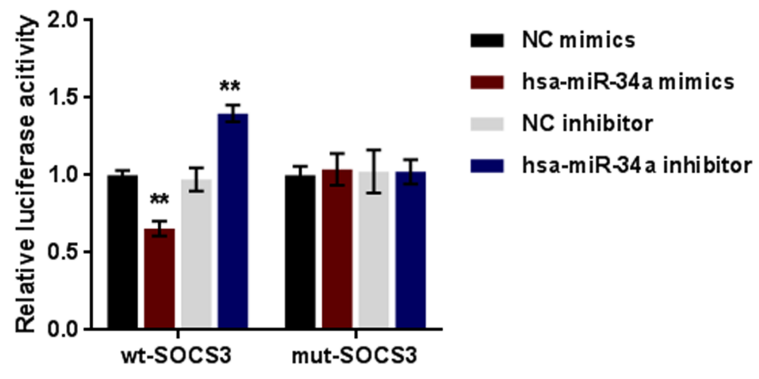

293T

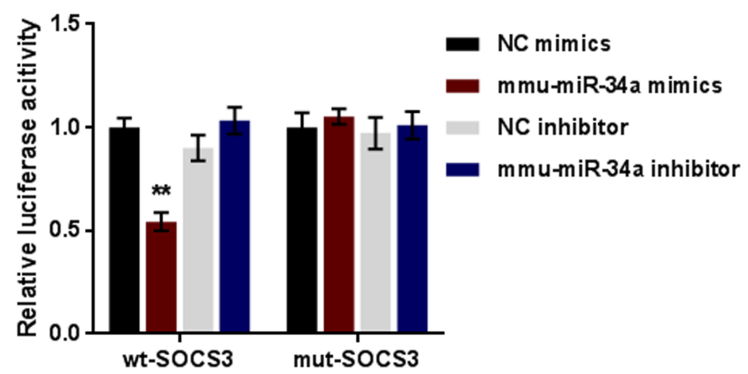

E
M1

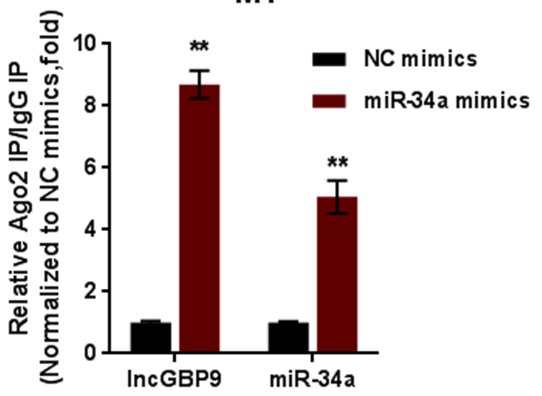

M1

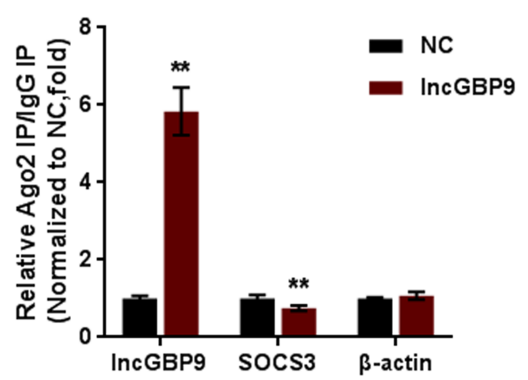

Figure 6 
LncGBP9 serves as a ceRNA for miR-34a to counteract miR-34a-mediated SOCS3 suppression (A-B) Schematic diagrams showing the predicted binding site between miR-34a and IncGBP9 and between miR-34a and SOCS3. Wild- and mutant-type GBP9 and SOCS3 3'-UTR luciferase reporter vectors (wtGBP9/SOCS3 3'-UTR or mut-GBP9/SOCS3 3'-UTR) were constructed and co-transfected in 293T cells with miR-34a mimics/inhibitor; the luciferase activity was determined. (C) Association of miR-34a and IncGBP9 with AGO2 in M1 macrophages. Detection of AGO2 and IgG using Immunoblotting assays. (D) RIP assay in M1 macrophages transfected with control miRNA (NC mimics) or miR-34a mimics followed by real-time PCR to detect GBP9 and miR-34a associated with AGO2. (E) RIP assay in M1 macrophages transfected with control vector (NC) or IncGBP9-overexpressing vector followed by real-time PCR to detect GBP9, SOCS3, and $\beta$-actin associated with $A G 02$. ${ }^{*} P<0.05, * \star P<0.01$. Values are mean $\pm S . D$ of $n=3$ independent experiments.

A

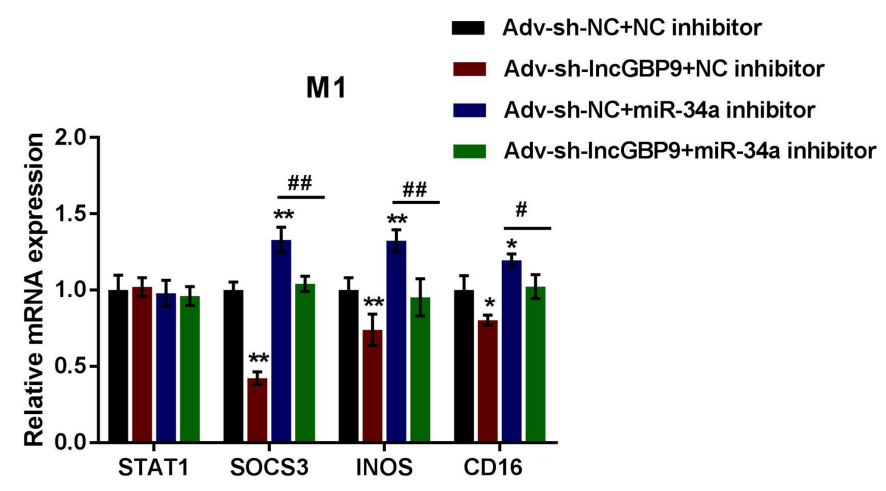

C

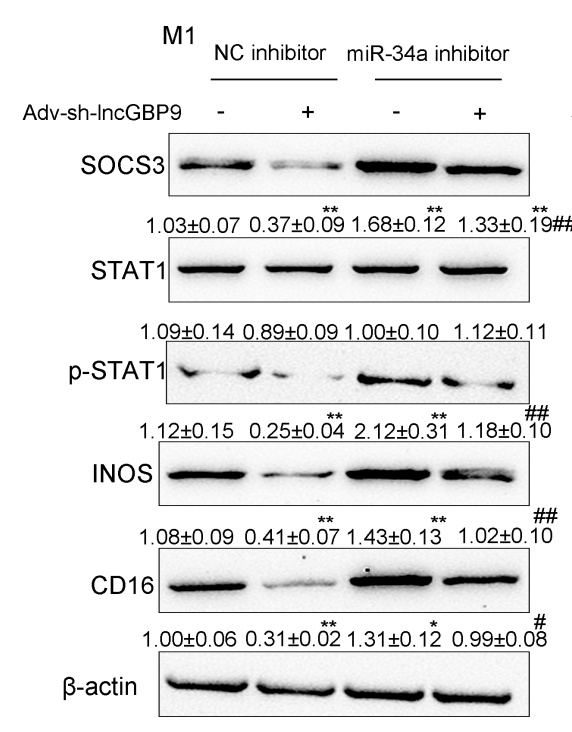

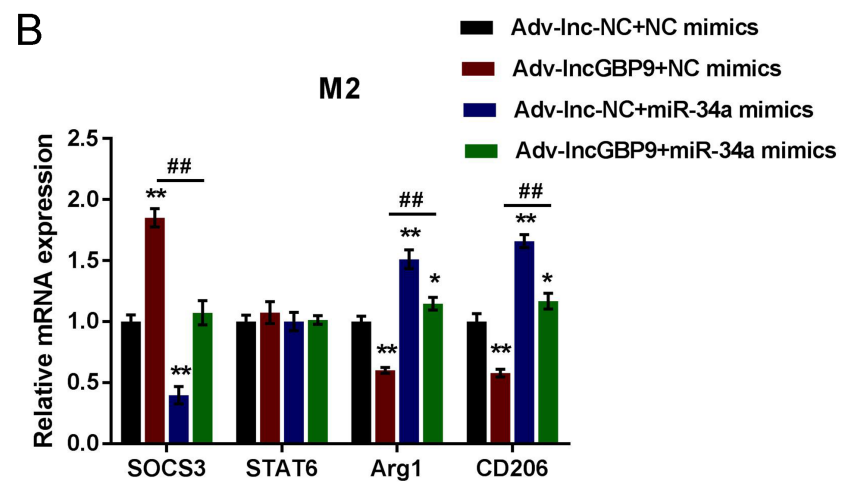
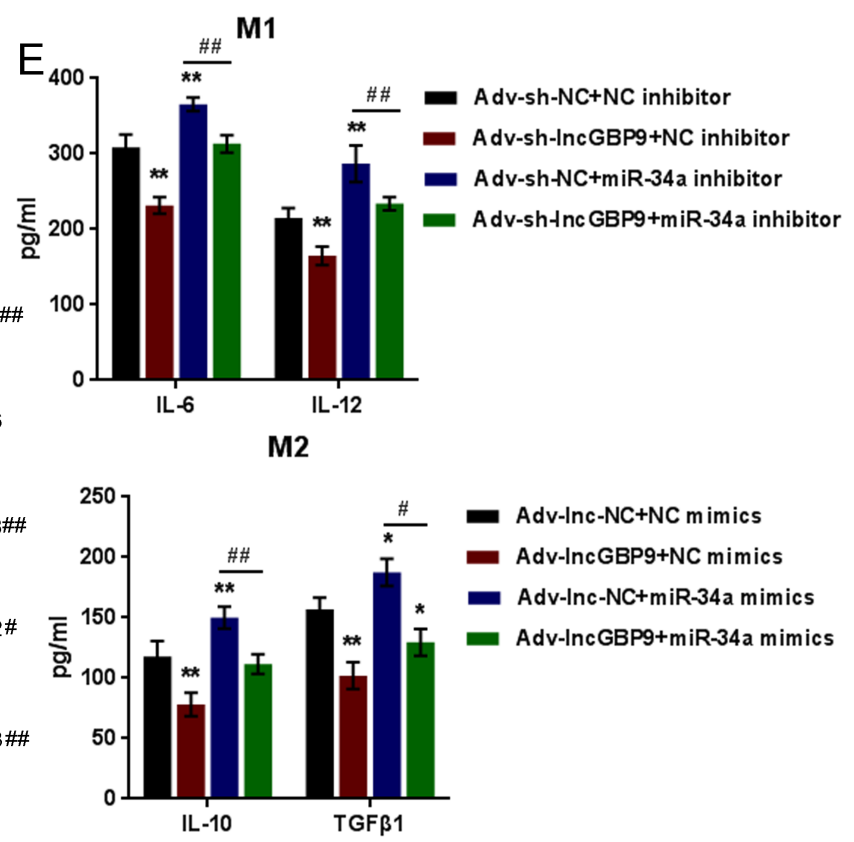

Figure 7

LncGBP9/miR-34a axis modulates macrophage polarization via affecting the balance of STAT1/STAT6 (A, C, and E) M1 macrophages were co-transfected with Ad-sh-IncGBP9 and miR-34a inhibitor and examined for the mRNA expression and protein levels of STAT1, p-STAT1, SOCS3, iNOS, and CD16/32, and the production of IL- 6 and IL-12. (B, D, and E) M2 macrophages were co-transfected with Ad-IncGBP9 
and miR-34a mimics and examined for the mRNA expression and protein levels of STAT6, p-STAT6, SOCS3, Arg1, and CD206, and the production of IL-10 and TGF- $\beta 1$. ${ }^{*} \mathrm{P}<0.05,{ }^{*} \mathrm{P}<0.01$, compared to control group; \#P<0.05, \#\#P<0.01, compared to Ad-sh-NC + miR-34a inhibitor or Ad-Inc-NC + miR-34a mimics group. Values are mean \pm S.D of $n=3$ independent experiments.

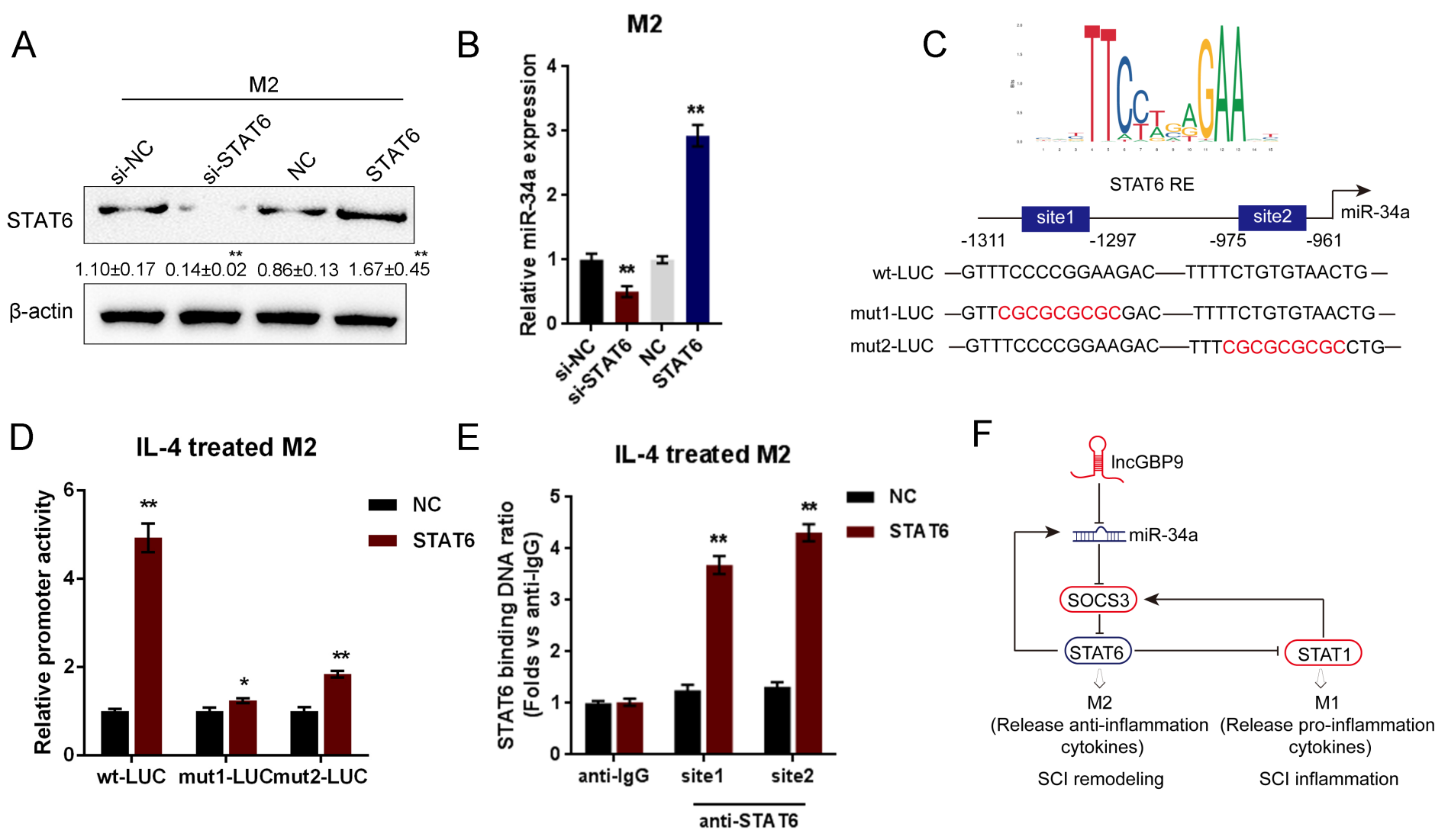

\section{Figure 8}

STAT6 binds miR-34a promoter to activate its transcription (A) STAT6 overexpression or silencing conducted in M2 macrophages by transfection of STAT6-overexpressing or si-STAT6 vector into M0 and then polarizing to M2, as confirmed by Immunoblotting. (B) The expression of miR-34a in STAT6overexpressing or STAT6-silenced M2 macrophages determined by real-time PCR. (C) A schematic diagram showing the predicted binding sites between STAT6 and miR-34a promoter. Wild- and mutanttype miR-34a luciferase reporter vectors are constructed. (D) STAT6 and wt- or mut-miR-34a were cotransfected in M0 macrophages followed M2 plorization; the luciferase activity was determined. (E) The real-time ChIP assay showed that the level of STAT6 antibody binding to miR-34a promoter was much greater than that of IgG. (F) In macrophages, IncGBP9 competed with SOCS3 for miR-34a binding to counteract miR-34a-mediated suppression on SOCS3, therefore modulating STAT1/STAT6 signaling and the polarization of macrophages. STAT6 bound the promoter of miR-34a to activate its transcription, therefore forming two different regulatory loops to modulate the polarization of macrophages after SCI. Values are mean \pm S.D of $n=3$ independent experiments.

\section{Supplementary Files}


This is a list of supplementary files associated with this preprint. Click to download.

- tableS1.docx

- Fig.S1.tif

- FIGs2.tif 